\title{
PSEUDOHERMITIAN IMMERSIONS, PSEUDO-EINSTEIN STRUCTURES, AND THE LEE CLASS OF A CR MANIFOLD
}

\author{
ElisabetTa Barletta AND Sorin Dragomir
}

Any nondegenerate $C R$ manifold carrying a fixed contact 1-form is known to possess (cf. N. Tanaka [T], S. Webster [W1]) a canonical linear connection (the Tanaka-Webster connection) parallelizing the Levi form and the maximal complex structure. This leads to an (already widely exploited, cf. D. Jerison \& J.M. Lee [JL1], [JL2], J. M. Lee [L1], [L2], H. Urakawa [U1], [U2], etc.) analogy between $\mathrm{CR}$ geometry on one hand, and both Hermitian and conformal geometry on the other.

To describe our point of view, let $M$ and $A$ be two CR manifolds of $\mathrm{CR}$ dimensions $n$ and $N=n+k, k \geqq 1$, respectively. A $C R$ immersion $f: M \rightarrow A$ is an immersion and a $C R$ map. If $f$ is the inclusion then $M$ is a $C R$ submanifold of $A$ (a $C R$ hypersurface when $k=1$ ). For instance, let $M^{2 n+1}$ be the intersection between the sphere $S^{2 n+3}$ and a transverse complex hypersurface in $C^{n+2}$. Then $M^{2 n+1}$ is a CR hypersurface of $S^{2 n+3}$ (in particular $M^{2 n+1}$ is strictly pseudoconvex). Let $M$ be a CR submanifold of $A$. Then $M$ is rigid in $A$ if any CR diffeomorphism $F: M \rightarrow M^{\prime}$ onto another CR submanifold $M^{\prime}$ of $A$ (e.g. $F$ may be the restriction of a biholomorphic mapping) extends to a $\mathrm{CR}$ automorphism of $A$ (e.g. if $A=S^{2 n+3}$ then $F$ should extend to a fractional linear, or projective, transformation preserving $S^{2 n+3}$ ). A theory of $\mathrm{CR}$ immersions has been initiated by S. Webster [W2]. There it is shown that $S^{2 n+1}$ is rigid in $S^{2 n+3}$ if $n \geqq 2$. Also, if $n \geqq 3$ then any CR hypersurface of $S^{2 n+3}$ is rigid. The basic idea in [W2] is to endow the ambient space $S^{2 n+3}$ with the TanakaWebster connection (rather than the Levi-Civita connection associated with the canonical Riemannian structure) and obtain CR analogues of the Gauss-Weingarten (respectively Gauss-Ricci-Codazzi) equations (from the theory of isometric immersions between Riemannian manifolds). In the end, these could be used to show that the intrinsic geometry determines the (CR analogue of the) second fundamental form of the given $\mathrm{CR}$ immersion. The main inconvenience of this approach seems to be the nonuniqueness of choice of a canonical connection on the CR submanifold (i.e. the induced and the 'intrinsic' Tanaka-Webster connections of the submanifold do not coincide, in general). In [D1] we compensate

Received September 28, 1994, revised April 7, 1995. 
for this inadequacy by restricting ourselves to a smaller class of $\mathrm{CR}$ immersions, as follows. Let $f: M \rightarrow A$ be a $\mathrm{CR}$ immersion between two strictly pseudoconvex CR manifolds on which contact 1-forms $\theta$ and $\Theta$ have been fixed. Then $f * \Theta=\lambda \theta$ for some $C^{\infty}$ function $\lambda: M \rightarrow R$. If $\lambda \equiv 1$ then $f$ is called isopseudohermitian (following the terminology in $[\mathrm{J}]$ ). An isopseudohermitian immersion $f: M \rightarrow A$ is a pseudohermitian ımmersion if $f(M)$ is tangent to the characteristic direction of $(A, \Theta)$. If this is the case then (by a result in [D1]) $f$ is an isometry (with respect to the Webster metrics of $(M, \theta)$ and $(A, \Theta)$ ). Also one may use the axiomatic description (of the Tanaka-Webster connection) in [T] to show that the induced and intrinsic connections on $M$ coincide. Moreover, by a result of $\mathrm{H}$. Urakawa (any CR map $f: M \rightarrow A$ satisfying $f_{*} T=\lambda T_{A}$ for some $\lambda \in C^{\infty}(M)$ with $T(\lambda)=0$ is harmonic with respect to the Webster metrics of $M$ and $A$, cf. Corollary 3.2 in [U3], p. 236) any pseudohermitian immersion is actually minimal. Cf. also Theorem 7 in [D1]. The present note is an application of this theory in connection with the problem of the existence of pseudo-Einstein pseudohermitian structures (i.e. for which the pseudohermitian Ricci tensor of the Tanaka-Webster connection is proportional to the Levi form, cf. J. M. Lee, [L1]) on (locally realizable) CR manifolds. As in [D1], our main tool consists of pseudohermitian analogues of the Gauss and Weingarten equations. In particular, we introduce the concept of normal Tanaka-Webster connection $\nabla^{\perp}$ (of a given pseudohermitian immersion between two strictly pseudoconvex CR manifolds). When $\nabla^{\perp}$ is flat we use the (pseudohermitian analogues of the) Gauss-Ricci-Codazzi equations to relate the pseudohermitian Ricci tensors of the Tanaka-Webster connections of the submanifold and ambient space (cf. Theorem 2). As a corollary, we may regard the Lee class $\gamma(M)$ (a cohomology class in the first cohomology group of the given (locally realizable) CR manifold $M$ with coefficients in the sheaf of CR-pluriharmonic functions [L1]) as an obstruction toward the existence of pseudohermitian immersions $f: M \rightarrow S^{2 N+1}$ with a flat normal Tanaka-Webster connection of a strictly pseudoconvex CR manifold $M$ in an odd dimensional sphere. Our methods are similar to those in B.Y. Chen \& H.S. Lue [CL] (where holomorphic immersions between Kaehler manifolds are dealt with). We exploit the symmetries of the curvature tensor field of the Tanaka-Webster connection (rather than the Riemannian-Christoffel tensor field in $[\mathrm{CL}]$ ) and deal with the highly complicated character (due to the presence of torsion terms there) of the Bianchi identities (cf. e.g. (40)). The key points (leading from (52) to (26) in Theorem 2) are Lemma 2 (the $(0,2)$-tensor field $E_{a}$ is proportional to the Levi form of the submanifold) and a nontrivial cancellation of torsion terms.

As a byproduct of the considerations in section 6 we show (cf. Theorem 4) the nonexistence of pseudohermitian immersions of $H_{n}(s)$ (a quotient of the Heisenberg group by a discrete group of dilations, carrying the contact form discovered in [D2] in analogy with the Boothby metric of a complex Hopf manifold, cf. [D3]) into a Tanaka-Webster flat strictly pseudoconvex CR manifold (e.g. $H_{N}$ or $U_{\alpha, \beta}$ ). The extension to which one may exploit the analogy 
with the case of holomorphic immersions in [CL] (cf. also our Theorem 3) is demonstrated at the close of the same section.

Several examples of $\mathrm{CR}$ immersions are examinated in section 9. In particular, CR immersions

$$
\partial D_{\left(\alpha_{1}, \cdots, \alpha_{n}\right), \beta} \rightarrow \partial D_{\left(\alpha_{1}, \cdots, \alpha_{N}\right), \beta},
$$

(between boundaries of pseudo-Siegel domains in $C^{n+1}$ and $C^{N+1}$ respectively) arise when looking at the weak pseudoconvexity locus of $D_{\alpha, \beta}=\left\{(z, w) \in C^{N+1}\right.$ : $\left.\sum_{j=1}^{N}\left|z_{\jmath}\right|^{2 \alpha} \jmath+\operatorname{Im}\left(w^{\beta}\right)-1<0\right\}$, cf. [BP]. The authors are grateful to the referee for drawing their attention upon the works by $\mathrm{H}$. Urakawa and for suggestions which improved the first version of the present paper.

\section{Definitions and basic formulae}

Let $M$ be a real $(2 n+1)$-dimensional $C^{\infty}$ manifold. A $C R$ structure (of $\mathrm{CR}$ dimension $n$ ) on $M$ is a complex subbundle $T_{1,0}(M)$, of complex rank $n$, of the complexified tangent bundle $C T M=T(M) \otimes C$ so that

$$
T_{1,0}(M) \cap T_{0,1}(M)=(0),
$$

and

$$
\left[\Gamma^{\infty}\left(T_{1,0}(M)\right), \Gamma^{\infty}\left(T_{1,0}(M)\right)\right] \leqq \Gamma^{\infty}\left(T_{1,0}(M)\right) .
$$

Here $T_{0,1}(M)=\overline{T_{1,0}(M)}$ (throughout an overbar denotes complex conjugation). Also, if $E \rightarrow M$ is a vector bundle over $M$ then $\Gamma^{\infty}(E)$ denotes the module of $C^{\infty}$ cross-section in $E$ (defined on some open set $U \subseteq M$, to be understood from the context) and $E_{x}$ is the fibre in $E$ over $x \in M$. A pair $\left(M, T_{1,0}(M)\right)$ is a $C R$ manifold (of $\mathrm{CR}$ dimension $n$ ). Its Levi distribution

$$
H(M)=\operatorname{Re}\left\{T_{1,0}(M) \oplus T_{0,1}(M)\right\}
$$

carries the complex structure $J: H(M) \rightarrow H(M)$ given by

$$
J(Z+\bar{Z})=i(Z-\bar{Z}),
$$

for any $Z \in T_{1,0}(M)$. Here $i=\sqrt{-1}$. Let $K \subset T^{*}(M)$ be the annihilator of $H(M)$, i.e. $K_{x}=\left\{\omega \in T_{x}^{*}(M): \operatorname{Ker}(\omega) \supseteqq H(M)_{x}\right\}$ for any $x \in M$. Then $K \rightarrow M$ is a real line subbundle of $T *(M)$. Assume from now on that $M$ is orientable. Then $K$ admits globally defined nowhere zero sections $\theta \in \Gamma^{\infty}(K)$ each of which is referred to as a pseudohermitian structure on $M$. The Levi form $G_{\theta}$ of $\left(M, T_{1,0}(M), \theta\right)$ is given by

$$
G_{\theta}(X, Y)=d \theta(X, J Y),
$$

for any $X, Y \in \Gamma^{\infty}(H(M))$ and $\left(M, T_{1,0}(M)\right)$ is nondegenerate if $G_{\theta}$ is nondegenerate for some $\theta \in \Gamma^{\infty}(K)$ (and thus for all). If $\left(M, T_{1,0}(M)\right.$ ) is non- 
degenerate and a pseudohermitian structure $\theta$ has been fixed, then there is a unique globally defined nowhere zero tangent vector field $T$ (the characteristic direction of $d \theta$ ) on $M$ transverse to $H(M)$ and satisfying

$$
T\lrcorner \theta=1, T\lrcorner d \theta=0 .
$$

Here $X\rfloor$ denotes the interior product with the tangent vector field $X$. Clearly

$$
T(M)=H(M) \oplus \boldsymbol{R} T .
$$

Therefore one may extend $J$ to a bundle morphism $J: T(M) \rightarrow T(M)$ by requesting that $J T=0$. Also, let $g_{\theta}$ be the Webster metric, i.e. the semi-Riemannian metric given by $g_{\theta}(X, Y)=G_{\theta}(X, Y), g_{\theta}(X, T)=0$ and $g_{\theta}(T, T)=1$ for any $X, Y \in H(M)$. The CR manifold $\left(M, T_{1,0}(M)\right)$ is strictly pseudoconvex if $G_{\theta}$ is positive definite for some $\theta \in \Gamma^{\infty}(K)$. If this is the case then $g_{\theta}$ is a Riemannian metric and, as it has been pointed out elsewhere (cf. e.g. [D1]) the synthetic object $\left(J, T, \theta, g_{\theta}\right)$ is a contact metric structure on $M$ (in the sense of D.E. Blair [B], p. 25). In general $\left(J, T, \theta, g_{\theta}\right)$ is not normal, and the obstruction to normality is the pseudohermitian torsion, a fragment of the torsion field of the Tanaka-Webster connection which we now recall. Cf. [T], [W1], any nondegenerate $\mathrm{CR}$ manifold $M$ on which a pseudohermitian structure has been specified carries a canonical linear connection $\nabla$ satisfying the following axioms :

i) $H(M)$ is parallel with respect to $\nabla$,

ii) $\nabla J=0$,

iii) $\nabla g_{\theta}=0$,

iv) $\pi_{+} \operatorname{Tor}(Z, W)=0$ for any $Z \in T_{1,0}(M), W \in C T M$,

where $\pi_{+}: C T M \rightarrow T_{1,0}(M)$ is the natural projection associated with the direct sum decomposition :

$$
C T M=T_{1,0}(M) \oplus T_{0,1}(M) \oplus C T,
$$

and Tor is the torsion tensor field of $\nabla$. The pseudohermitian torsion $\tau$ of the Tanaka-Webster connection is the vector bundle valued 1 -form on $M$ given by

$$
\tau X=\operatorname{Tor}(T, X),
$$

for any $X \in H(M)$. Cf. [D1], $\operatorname{trace}(\tau)=0$ and $\tau$ is self-adjoint with respect to the Webster metric $g_{\theta}$. Also $\left(J, T, \theta, g_{\theta}\right)$ in normal (in the sense of [B], p. 48) iff $\tau=0$.

Let $\left(M, T_{1,0}(M), \theta\right)$ be nondegenerate and let $\nabla^{\theta}$ be the Levi-Civita connection of $\left(M, g_{\theta}\right)$. Then

$$
\nabla^{\theta}=\nabla+\left(\frac{1}{2} \Omega_{\theta}-A\right) \otimes T+\tau \otimes \theta+\theta \odot J .
$$

Here $\Omega_{\theta}(X, Y)=g_{\theta}(X, J Y)$ and $A(X, Y)=g_{\theta}(\tau X, Y)$ for any $X, Y \in H(M)$. Also $\odot$ denotes the symmetric product (e.g. $(\theta \odot J)(X, Y)=1 / 2\{\theta(X) J Y+\theta(Y) J X\})$. 
Furthermore, we shall need the identities

$$
\begin{aligned}
& \text { Tor }=2 \theta \wedge \tau-\Omega_{\theta} \otimes T, \\
& \nabla T=0, \\
& \tau J+J \tau=0 .
\end{aligned}
$$

Cf. [T]. Let $R$ be the curvature tensor field of the Tanaka-Webster connection $\nabla$ of $(M, \theta)$. Let $\operatorname{Ric}(X, Y)=\operatorname{trace}\{Z \mapsto R(Z, X) Y\}$ for any tangent vector fields $X, Y$ on $M$. If $\left\{T_{1}, \cdots, T_{n}\right\}$ is a (local) frame of $T_{1,0}(M)$, the pseudohermitian Ricci tensor $R_{\alpha, \bar{\beta}}$ of $(M, \theta)$ is given by

where $T_{\bar{\alpha}}=\bar{T}_{\alpha}$. Set also

$$
R_{\alpha \bar{\beta}}=\operatorname{Ric}\left(T_{\alpha}, T_{\bar{\beta}}\right),
$$

$$
h_{\alpha \bar{\beta}}=G_{\theta}\left(T_{\alpha}, T_{\bar{\beta}}\right) .
$$

Then $\theta$ is (globally) pseudo-Einstein if

$$
R_{\alpha \bar{\beta}}=\lambda h_{\alpha \bar{\beta}},
$$

for some $C^{\infty}$ function $\lambda$, i.e. the pseudohermitian Ricci tensor of $(M, \theta)$ is proportional to the Levi form (cf. [L1]). If this is the case then $\lambda=(1 / n) R$ where $R=h^{\alpha \bar{\beta}} R_{\alpha \bar{\beta}}$ is the pseudohermitian scalar curvature of $(M, \theta)$. The pseudo-Einstein condition (13) is not so rigid as its Riemannian counterpart. Indeed, the II nd Bianchi identity (associated with the Tanaka-Webster connection) no longer implies $R=$ const. (due to the presence of torsion terms). It should also be pointed out that (unlike the case of Kaehler geometry) $R_{\alpha \bar{\beta}}$ is only a fragment of Ric (Ric is determined by $R_{\alpha \bar{\beta}}$ and certain covariant derivatives of $\tau$, cf. [D1]). Any odd dimensional sphere $S^{2 n+1} \subset C^{n+1}$ endowed with the standard CR structure $T_{1,0}\left(S^{2 n+1}\right)=T^{1,0}\left(\boldsymbol{C}^{n+1}\right) \cap C T S^{2 n+1}$ admits the pseudoEinstein pseudohermitian structure $\iota^{*} \theta$ where $\theta=i / 2(\bar{\partial}-\partial)|z|^{2}$ and $\iota: S^{2 n+1} \subset C^{n+1}$. Throughout, if $X$ is a complex manifold, then $T^{1,0}(X)$ denotes its holomorphic tangent bundle. Also, if $H_{n}=\boldsymbol{C}^{n} \times \boldsymbol{R}$ is the Heisenberg group (cf. e.g. [FS], p. 434-435) and $\boldsymbol{\delta}_{s}: H_{n}-\{0\} \rightarrow H_{n}-\{0\}$ the dilation by $0<s<1$ then $G_{s}=\left\{\boldsymbol{\delta}_{s}^{m}: m \in \boldsymbol{Z}\right\}$ acts freely on $H_{n}-\{0\}$ as a properly discontinuous group of CR automorphisms of $H_{n}-\{0\}$ so that (cf. [D2], p. 36) the quotient space $H_{n}(s)=\left(H_{n}-\{0\}\right) / G_{s}$ is a compact $\mathrm{CR}$ manifold (of CR dimension $n$ ) diffeomorphic to $\Sigma^{2 n} \times S^{1}$, where $\Sigma^{2 n}=\left\{x \in H_{n}:|x|=1\right\}$ and $|x|=\left(|z|^{4}+t^{2}\right)^{1 / 4}$ is the Heisenberg norm of $x=(z, t)$. By a result in [D2] the pseudohermitian structure $\theta=|x|^{-2}\left\{d t+2 \sum_{\alpha=1}^{n}\left(x^{\alpha} d y^{\alpha}-\right.\right.$ $\left.\left.y^{\alpha} d x^{\alpha}\right)\right\}$ on $H_{n}(s)$ is pseudo-Einstein (here $\left.x=(z, t), z=\left(z^{1}, \cdots, z^{n}\right), z^{\alpha}=x^{\alpha}+i y^{\alpha}\right)$.

\section{Pseudohermitian immersions}

Let $\left(M, T_{1,0}(M)\right)$ and $\left(A, T_{1,0}(A)\right)$ be two $\mathrm{CR}$ manifolds of CR dimensions $n$ and $N=n+k$, respectively. A $C^{\infty}$ map $f: M \rightarrow A$ is a $C R$ map if 
PSEUDOHERMITIAN IMMERSIONS, PSEUDO-EINSTEIN STRUCTURES, LEE CLASS 67

$$
f_{*} T_{1,0}(M) \subset T_{1,0}(A) .
$$

Let us assume from now on that $\left(M, T_{1,0}(M)\right)$ and $\left(A, T_{1,0}(A)\right)$ are strictly pseudoconvex and specify pseudohermitian structures $\theta$ and $\Theta$, on $M$ and $A$ respectively, so that $G_{\theta}$ and $G_{\Theta}$ are positive definite. Let $f: M \rightarrow A$ be a CR map. Then

$$
f * \Theta=\mu \theta,
$$

for some $C^{\infty}$ function $\mu>0$. Assume from now on that $f$ is a CR immersion (i.e. an immersion and a $C R$ map). A theory of $C R$ immersions (between strictly pseudoconvex CR manifolds) has been built in [D1]. We recall that in general $f$ is not an isometry with respect to the Webster metrics $g_{\theta}$ and $g_{\theta}$. Yet, if $f$ is isopseudohermitian (i.e. $\mu=1$ ), then $f^{*} g_{\theta}=g_{\theta}$ iff $\operatorname{nor}\left(T_{A}\right)=0$. Here $T_{A}$ is the characteristic direction of $d \Theta$ and nor $_{x}: T_{f(x)}(A) \rightarrow \nu^{2 k}(f)_{x}$ is the natural projection with respect to the direct sum decomposition

$$
T_{f(x)}(A)=\left[\left(d_{x} f\right) T_{x}(M)\right] \oplus \nu^{2 k}(f)_{x},
$$

for any $x \in M$. Here $\nu^{2 k}(f) \rightarrow M$ denotes the normal bundle of the given immersion. Such $f: M \rightarrow A$ (i.e. a CR immersion with $f * \Theta=\theta$ and $\operatorname{nor}\left(T_{A}\right)=0$ ) is termed pseudohermitian immersion. If this is the case then $f_{*} T=T_{A}$. Also (cf. [D1]) there are natural CR analogues of the Gauss and Weingarten formulae

$$
\begin{aligned}
& \nabla_{f * X}^{A} f_{*} Y=f_{*} \nabla_{X} Y+\alpha(f)(X, Y), \\
& \nabla_{f * X}^{A} \xi=-f_{*} a_{\xi} X+\nabla_{X}^{\perp} \xi,
\end{aligned}
$$

for any $X, Y \in \Gamma^{\infty}(T M), \xi \in \Gamma^{\infty}\left(\nu^{2 k}(f)\right)$. Here $\nabla, \nabla^{A}$ are the Tanaka-Webster connections of $(M, \theta),(A, \Theta)$, respectively. Also $\alpha(f)$ and $a$ are bilinear and $\nabla^{\perp}$ is a connection in $\nu^{2 k}(f)$, referred to as the normal Tanaka-Webster connection of $f$. Unlike the second fundamental form of $f$, its CR analogue $\alpha(f)$ is not symmetric, i.e.

$$
\alpha(f)(X, Y)-\alpha(f)(Y, X)=\text { nor }\left\{\operatorname{Tor}_{A}\left(f_{*} X, f_{*} Y\right)\right\},
$$

for any $X, Y \in T(M)$. Here $\operatorname{Tor}_{A}$ is the torsion tensor field of $\nabla^{A}$. Since $\nabla^{A} T_{A}=0$ it follows that

$$
\alpha(f)(X, T)=0,
$$

for any $X \in T(M)$. We consider the normal bundle valued 1-form $Q(f)$ on $M$ given by

$$
Q(f) X=\alpha(f)(T, X),
$$

for any $X \in T(M)$. If $\tau_{A}$ is the pseudohermitian torsion of the Tanaka-Webster connection $\nabla^{A}$ then

$$
\tau_{A} f_{*} X=f_{*} \tau X+Q(f) X .
$$

Taking into account (10) and (17), the identity (16) may be also written 


$$
\alpha(f)(X, Y)-\alpha(f)(Y, X)=2(\theta \wedge Q f)(X, Y),
$$

for any $X, Y \in T(M)$. The equations (14)-(15) lead to $\mathrm{CR}$ analogues of the Gauss-Codazzi-Ricci equations

$$
\begin{aligned}
& \tan \left\{R^{A}\left(f_{*} X, f_{*} Y\right) f_{*} Z\right\}=R(X, Y) Z+a_{\alpha(f)(X, Z)} Y-a_{\alpha(f)(Y, Z)} X, \\
& \operatorname{nor}\left\{R^{A}\left(f_{*} X, f_{*} Y\right) f_{*} Z\right\} \\
= & \left(\nabla_{X} \alpha(f)\right)(Y, Z)-\left(\nabla_{Y} \alpha(f)\right)(X, Z)+\alpha(f)(\operatorname{Tor}(X, Y), Z), \\
& g_{\theta}\left(R^{A}\left(f_{*} X, f_{*} Y\right) \xi, \eta\right) \\
= & g_{\theta}\left(R^{\perp}(X, Y) \xi, \eta\right)+g_{\theta}\left(a_{\eta} Y, a_{\xi} X\right)-g_{\theta}\left(a_{\eta} X, a_{\xi} Y\right),
\end{aligned}
$$

for any $X, Y, Z \in T(M)$ and any $\xi, \eta \in \nu^{2 k}(f)$. Here $\tan _{x}: T_{f(x)}(A) \rightarrow T_{x} M$ is the natural projection, $x \in M$, and $R^{A}, R^{\perp}$ are the curvature tensor fields of $\nabla^{A}$, $\nabla^{\perp}$, respectively. Note that

$$
g_{\theta}\left(a_{\xi} X, Y\right)=g_{\theta}(\alpha(f)(X, Y), \xi) .
$$

Therefore (on account of (16)), unlike the Weingarten operator of $f$, its CR analogue $a_{\xi}$ is not self-adjoint (unless $Q(f)=0$ ). Also (by $(21)$ ) $a_{\xi}$ is $H(M)$ valued. As $f$ is a $C R$ map

$$
\begin{aligned}
& f_{*} H(M) \subset H(A), \\
& f_{*} \circ J=J_{A^{\circ}} f_{*},
\end{aligned}
$$

where $J_{A}: H(A) \rightarrow H(A)$ denotes the complex structure of $H(A)$. Next $\nabla^{A} J_{A}=0$ and (14)-(15) yield

$$
\begin{aligned}
& \alpha(f)(X, J Y)=J_{A} \alpha(f)(X, Y), \\
& a_{J_{A} \xi} X=J a_{\xi} X, \\
& \nabla^{\perp} J_{A}=0,
\end{aligned}
$$

for any $X, Y \in T(M), \xi \in \nu^{2 k}(f)$. Cf. [D1], $f^{*} g_{\theta}=g_{\theta}$ yields $\nu^{2 k}(f) \subset H(A)$ so that $J_{A} \xi$ makes sense $a$ priori (i.e. before the extension of $J_{A}$ to a $(1,1)$-tensor field on $A$ by requesting that $\left.J_{A} T_{A}=0\right)$. Conversely, if $\nu_{H}^{2 k}(f)_{x}$ is the orthogonal complement (with respect to the inner product $\left.g_{\theta, f(x)}\right)$ of $\left(d_{x} f\right) H(M)_{x}$ in $H(A)_{f(x)}$ and $\nu_{H}^{2 k}(f)_{x}=\nu^{2 k}(f)_{x}$ for any $x \in M$, then $f$ is an isometry with respect to the Webster metrics of $(M, \theta)$ and $(A, \Theta)$.

\section{CR-pluriharmonic functions and the Lee class}

Let $M \subset C^{n+1}$ be a real hypersurface. Then $T_{1,0}(M)=T^{1,0}\left(\boldsymbol{C}^{n+1}\right) \cap \boldsymbol{C T M}$ is a CR structure of CR dimension $n$ on $M$. Such $\left(M, T_{1,0}(M)\right)$ is referred to as an embedded CR manifold. A CR manifold $\left(M, T_{1,0}(M)\right)$ is locally realizable if 
each point of $M$ admits a neighborhood which is $\mathrm{CR}$ isomorphic to some embedded CR manifold. If $\left(M, T_{1,0}(M)\right)$ is a locally realizable CR manifold then pseudoEinstein pseudohermitian structures exist (locally) in some neighborhood of every point of $M$ (cf. Corollary B of [L1]) but there may be obstructions to the existence of global pseudo-Einstein structures. Let $\mathscr{P}$ be the sheaf of $C R$-pluriharmonic functions on $M$, i.e. if $U \subseteq M$ is open then $u \in \mathscr{Q}(U)$ iff $u=\operatorname{Re}(F)$ for some CR-holomorphic function $F: U \rightarrow C$. Then there is a CR-invariant cohomology class $\gamma(M) \in H^{1}(M, \mathscr{Q})$ (referred hereafter as the Lee class of $M$ ) which vanishes iff $M$ admits a global pseudo-Einstein structure (cf. [L1], p. 172). A complex valued $q$-form $\eta$ on $M$ is a $(0, q)$-form if $T\lrcorner \eta=0$ and $\left.T_{1,0}(M)\right\rfloor \eta=0$. For instance, if $\left\{\theta^{1}, \cdots, \theta^{n}\right\}$ is an admissible coframe, i.e. $\theta^{\alpha}$ are the (local) 1-forms determined by $\left.\left.T_{\beta}\right\rfloor \theta^{\alpha}=\delta_{\beta}^{\alpha}, T\right\lrcorner \theta^{\alpha}=0$ and $\left.T_{\bar{\beta}}\right\lrcorner \theta^{\alpha}=0$, then any $(0,1)$ form $\eta$ may be written locally as $\eta=\eta_{\bar{\alpha}} \theta^{\bar{\alpha}}$, where $\theta^{\bar{\alpha}}=\overline{\theta^{\alpha}}$. Let $\Lambda^{0, q}(M)$ be the bundle of $(0, q)$-forms on $M$. The tangential Cauchy-Riemann operator is the differential operator $\bar{\partial}_{M}: \Gamma^{\infty}\left(\Lambda^{0, q}(M)\right) \rightarrow \Gamma^{\infty}\left(\Lambda^{0, q+1}(M)\right)$ defined as follows. Let $\eta$ be a $(0, q)$-form on $M$. Then $\bar{\partial}_{M} \eta$ is the unique $(0, q+1)$-form which coincides with $d \eta$ when restricted to $T_{0,1}(M) \otimes \cdots \otimes T_{0,1}(M)(q+1$ factors). A $(0, q)$-form $\eta$ is $C R$-holomorphic if it satisfies the tangential Cauchy-Riemann equations

$$
\bar{\partial}_{M} \eta=0 \text {. }
$$

Let $f: M \rightarrow A$ be a pseudohermitian immersion. Then

$$
\bar{\partial}_{M} f * \eta=f * \bar{\partial}_{A} \eta,
$$

for any $(0, q)$-form $\eta$ on $A$. Let $\mathscr{P}_{A}$ be the sheaf of CR-pluriharmonic functions on $A$. Assume for the rest of this section that $f$ is a homeomorphism on its image. As a consequence of (25), if $D \cong A$ is open and $v \in \mathscr{P}_{A}(D)$ then $v \circ f \in \mathscr{P}(V)$, where $V=f^{-1}(D \cap f(M))$. We need to recall the construction of the CR-invariant cohomology class $\gamma(A) \in H^{1}\left(A, \mathscr{Q}_{A}\right)$ built in [L1], p. 172. Assume from now on that $A$ is locally realizable (e.g. if either $A$ is compact or $N>2$, then by results in L. Boutet De Monvel [BM] (for the compact case) and M. Kuranishi [K], T. Akahori [A] (for the noncompact case) it follows that $\left(A, T_{1,0}(A)\right.$ ) is locally realizable). Then, by a result in $[\mathrm{L} 1]$, p. 158, there is an open covering $\mathscr{D}=$ $\left\{D_{j}\right\}_{j \in \Sigma}$ of $A$ and a pseudo-Einstein pseudohermitian structure $\Theta_{\jmath}$ on each $D_{\jmath}$, $j \in \Sigma$. If $I_{\imath \jmath}: D_{i} \cap D_{j} \rightarrow D_{\jmath}$ are inclusions, then $I_{i j}^{*} \Theta_{\jmath}=\exp \left(2 U_{j i}\right) I_{j i}^{*} \Theta_{\imath}$ for some $C^{\infty}$ functions $U_{j i}: D_{i} \cap D_{j} \rightarrow \boldsymbol{R}$. By Proposition 5.1 of $[\mathrm{L} 1]$, p. $172, U_{j i} \in \mathscr{Q}_{A}\left(D_{i} \cap D_{\jmath}\right)$. Let $N(\mathscr{D})$ be the nerve of $\mathscr{D}$ (we use the notations and conventions in $\mathrm{S}$. Goldberg [G], p. 272-275). Let $C \in \mathcal{C}^{1}\left(N(\mathscr{D}), \mathscr{Q}_{A}\right)$ be the 1-cochain mapping each 1-simplex $\sigma=\left(D_{i} D_{j}\right)$ of $N(\mathscr{D})$ in $U_{j i} \in \mathscr{Q}_{A}(\cap \sigma)$. Then $C \in Z^{1}\left(N(\mathscr{D})\right.$, $\left.\mathscr{Q}_{A}\right)$, i.e. $C$ so built is a 1-cocycle with coefficients in $\mathscr{Q}_{A}$. Finally $\gamma(A) \in H^{1}\left(A, \mathscr{Q}_{A}\right)$ is the equivalence class of $[C] \in H^{1}\left(N(\mathscr{D}), \mathscr{Q}_{A}\right)$. Note that each pseudohermitian immersion $f: M \rightarrow A$ (so that $f: M \rightarrow f(M)$ is a homeomorphism) induces a map on cohomology $f^{*}: H^{p}\left(A, \mathscr{Q}_{A}\right) \rightarrow H^{p}(M, \mathscr{Q})$. Let $\operatorname{Cov}(A)$ be the set of all open coverings of $A$. Let $\Gamma \in H^{p}\left(A, \mathscr{Q}_{A}\right)$. Since 


$$
H^{p}\left(A, \mathscr{Q}_{A}\right)=\varliminf H^{p}\left(N(\mathscr{D}), \mathscr{Q}_{A}\right),
$$

there is $\mathscr{D} \in \operatorname{Cov}(A)$ and $h \in H^{p}\left(N(\mathscr{D}), \mathscr{Q}_{A}\right)$ so that $\Gamma=[h]$. Let $V_{j}=f^{-1}\left(D_{j} \cap f(M)\right)$ and set $\mathscr{V}=\left\{V_{j}\right\}_{j \in \Sigma}$. Then $\mathcal{V} \in \operatorname{Cov}(M)$. Set $f^{*} \Gamma=\left[f^{*} h\right]$ where $f^{*}$ : $H^{p}\left(N(\mathscr{D}), \mathscr{Q}_{A}\right) \rightarrow H^{p}(N(\mathcal{V}), \mathscr{P})$ is described as follows. Let $c \in Z^{p}\left(N(\mathscr{D}), \mathscr{P}_{A}\right)$ so that $h=[c]$ and set $f^{*} h=[f * c]$ where $f^{*}: \mathcal{C}^{p}\left(N(\mathscr{D}), \mathscr{P}_{A}\right) \rightarrow \mathcal{C}^{p}(N(\mathcal{C}), \mathscr{P})$ is described as follows. Let $\sigma=\left(V, \jmath_{0} \cdots V_{\jmath_{p}}\right)$ be a $p$-simplex of $N(C V)$ and set

$$
\left(f^{*} c\right) \sigma=\rho_{f * \sigma, \sigma} c\left(f_{*} \sigma\right),
$$

where $f^{*} \sigma=\left(D_{\jmath_{0}} \cdots D_{\jmath_{p}}\right)$ while $\rho_{f * \sigma, \sigma}: \mathscr{Q}_{A}\left(\cap f_{*} \sigma\right) \rightarrow \mathscr{Q}(\cap \sigma)$ is given by

$$
\rho_{f * \sigma, \sigma}(v)=v \circ f
$$

for any CR-pluriharmonic function $v: D_{\jmath_{0}} \cap \cdots \cap D_{\jmath_{p}} \rightarrow \boldsymbol{R}$. It is an elementary matter to check that the definition of $f^{*}$ doesn't depend (at the various stages) on the choice of representatives. We may state the following

THEOREM 1. Let $f: M \rightarrow A$ be a pseudohermitian immersion (so that $f$ : $M \rightarrow f(M)$ is a homeomorphism) between two strictly pseudoconvex $C R$ manifolds $M$ and $A$ of $C R$ dimensions $n$ and $N=n+k$. Assume that both $M, A$ are locally realizable (e.g. either $M, A$ are compact on $n>2$ ). Then

$$
f^{*} \gamma(A)-\gamma(M) \in \operatorname{Ker}(j),
$$

where $j: H^{1}(M, \mathscr{P}) \rightarrow H^{1}(M, \mathcal{E})$ is the map induced on cohomology by the natural sheaf morphism $\mathscr{P} \rightarrow \mathcal{E}$ (and $\mathcal{E}$ is the sheaf of $C^{\infty}$ functions on $M$ ). Set $\varphi_{j}=f * \Theta_{\jmath}$, $V_{\jmath}=f^{-1}\left(D_{j} \cap f(M)\right), j \in \Sigma$. If each $\left(V_{\jmath}, \varphi_{j}\right)$ is pseudo-Einstein then $f^{*} \gamma(A)=\gamma(M)$; in particular, if $A$ admits a global pseudo-Einstein structure, then so does $M$.

Given a pseudohermitian immersion, between two strictly pseudoconvex CR manifolds $(M, \theta)$ and $(A, \Theta)$ so that $\Theta$ is pseudo-Einstein, it is natural to ask (on account of Theorem 1) whether $\theta$ is pseudo-Einstein, as well. We obtain the following

THEOREM 2. Let $f: M \rightarrow A$ be a pseudohermitian immersion between two strictly pseudoconvex $C R$ manifolds $(M, \theta)$ and $(A, \Theta)$. If the normal TanakaWebster connection is flat (i.e. $R^{\perp}=0$ ) then

$$
R_{\alpha \bar{\beta}}=\operatorname{trace}\left\{Z \mapsto R^{A}\left(Z, f_{*} T_{\alpha}\right) f_{*} T_{\bar{\beta}}\right\} .
$$

In particular, if $\Theta$ is pseudo-Einstein then $\theta$ is pseudo-Einstein, too.

COROLLARY 1. Let $M \rightarrow S^{2 N+1}$ be a pseudohermitian immersion with a flat normal Tanaka-Webster connection, of a strictly pseudoconvex $C R$ manifold $M$ in the standard sphere. Then $\gamma(M)=0$. 
PSEUDOHERMITIAN IMMERSIONS, PSEUDO-EINSTEIN STRUCTURES, LEE CLASS 71

\section{Consequences of the embedding equations}

We shall need the following holds

LEMMA 1. For any $X, Y \in T(M)$ and any $\xi \in \nu^{2 k}(f)$ the following identity

$$
\begin{aligned}
g_{\theta}\left(a_{\xi} J X+J a_{\xi} X, Y\right)= & g_{\theta}\left(\operatorname{Tor}_{A}\left(f_{*} X, f_{*} Y\right), J_{A} \xi\right) \\
& +g_{\theta}\left(\operatorname{Tor}_{A}\left(f_{*} J X, f_{*} Y\right), \xi\right) .
\end{aligned}
$$

Proof. Using (21), (16), (22) and again (16) we may conduct the following calculation

Finally

$$
\begin{aligned}
g_{\theta}\left(a_{\xi} J X, Y\right)= & g_{\theta}(\alpha(f)(J X, Y), \xi) \\
= & g_{\Theta}\left(\alpha(f)(Y, J X)+\operatorname{Tor}_{A}\left(f_{*} J X, f_{*} Y\right), \xi\right) \\
= & g_{\theta}\left(J_{A} \alpha(f)(Y, X)+\operatorname{Tor}_{A}\left(f_{*} J X, f_{*} Y\right), \xi\right) \\
= & g_{\Theta}\left(J_{A} \alpha(f)(X, Y), \xi\right)+g_{\theta}\left(\operatorname{Tor}_{A}\left(f_{*} J X, f_{*} Y\right), \xi\right) \\
& -g_{\Theta}\left(J_{A} \operatorname{nor}\left\{\operatorname{Tor}_{A}\left(f_{*} X, f_{*} Y\right)\right\}, \xi\right) .
\end{aligned}
$$

$$
\begin{aligned}
& J_{A}^{2}=-I+\Theta \otimes T_{A}, \\
& g_{\Theta}\left(J_{A} X, J_{A} Y\right)=g_{\Theta}(X, Y)-\Theta(X) \Theta(Y),
\end{aligned}
$$

lead to (27).

Q.E.D. furnish

Let $\xi \in \nu^{2 k}(f)$ so that $R^{\perp}(X, Y) \xi=0$ for any $X, Y \in T(M)$. Then (20) and (23)

$$
R^{A}\left(f_{*} X, f_{*} Y ; \xi, J_{A} \xi\right)=g_{\theta}\left(J a_{\xi} Y, a_{\xi} X\right)-g_{\theta}\left(J a_{\xi} X, a_{\xi} Y\right) .
$$

Throughout $R(X, Y ; Z, W)=g_{\theta}(R(X, Y) Z, W)$, etc.. Note that (16) may be restated as

$$
g_{\theta}\left(a_{\xi} X, Y\right)=g_{\theta}\left(X, a_{\xi} Y\right)+g_{\theta}\left(\operatorname{Tor}_{A}\left(f_{*} X, f_{*} Y\right), \xi\right) .
$$

By (31) and $J^{2}=-I+\theta \otimes T$ we obtain

$$
g_{\theta}\left(J a_{\xi} Y, a_{\xi} X\right)=-g_{\theta}\left(a_{\xi} J a_{\xi} X, Y\right)-g_{\theta}\left(\operatorname{Tor}_{A}\left(f_{*} Y, f_{*} J a_{\xi} X\right), \xi\right) .
$$

Let us replace $X$ by $a_{\xi} X$ in (27) of Lemma 1 so that to yield

$$
\begin{aligned}
g_{\theta}\left(a_{\xi} J a_{\xi} X, Y\right)= & -g_{\theta}\left(J a_{\xi}^{2} X, Y\right)+g_{\theta}\left(\operatorname{Tor}_{A}\left(f_{*} a_{\xi} X, f_{*} Y\right), J_{A} \xi\right) \\
& +g_{\theta}\left(\operatorname{Tor}_{A}\left(f_{*} J a_{\xi} X, f_{*} Y\right), \xi\right) .
\end{aligned}
$$

Substitution from (33) into (32) now leads to 


$$
g_{\theta}\left(J a_{\xi} Y, a_{\xi} X\right)=g_{\theta}\left(J a_{\xi}^{2} X, Y\right)-g_{\theta}\left(\operatorname{Tor}_{A}\left(f_{*} a_{\xi} X, f_{*} Y\right), J_{A} \xi\right) .
$$

On the other hand we may replace $X$ by $Y$ and $Y$ by $J a_{\xi} X$ in (31). The resulting identity and (33) furnish

$$
g_{\theta}\left(J a_{\xi} X, a_{\xi} Y\right)=-g_{\theta}\left(J a_{\xi}^{2} X, Y\right)+g_{\theta}\left(\operatorname{Tor}_{A}\left(f_{*} a_{\xi} X, f_{*} Y\right), J_{A} \xi\right) .
$$

Finally, by (34)-(35) the (CR analogue of) Ricci's equation (30) becomes

$$
R^{A}\left(f_{*} X, f_{*} Y ; \xi, J_{A} \xi\right)=2 g_{\theta}\left(J a_{\xi}^{2} X, Y\right)-2 g_{\theta}\left(\operatorname{Tor}_{A}\left(f_{*} a_{\xi} X, f_{*} Y\right), J_{A} \xi\right),
$$

for any $X, Y \in T(M)$ and $\xi \in \nu^{2 k}(f)$ with the property $R^{\perp}(X, Y) \xi=0$. Let $\left\{\xi_{1}, \cdots, \xi_{k}, J_{A} \xi_{1}, \cdots, J_{A} \xi_{k}\right\}$ be a local orthonormal frame of $\nu^{2 k}(f)$ and $\left\{E_{1}, \cdots\right.$, $\left.E_{2 n+1}\right\}$ a local orthonormal frame of $T(M)$, with $E_{2 n+1}=T$ and $E_{j} \in H(M)$, $1 \leqq j \leqq 2 n$. Let $K(Z, W)=\operatorname{trace}\left\{V \mapsto R^{A}(V, Z) W\right\}$. It is our purpose of compute $K\left(f_{*} X, f_{*} Y\right)$ for any $X, Y \in T(M)$. To this end, note that (18) may be restated as follows

$$
\begin{aligned}
& R^{A}\left(f_{*} X, f_{*} Y ; f_{*} Z, f_{*} W\right) \\
= & R(X, Y ; Z, W)+g_{\theta}(\alpha(f)(Y, W), \alpha(f)(X, Z)) \\
& -g_{\theta}(\alpha(f)(X, W), \alpha(f)(Y, Z)),
\end{aligned}
$$

for any $X, Y, Z, W \in T(M)$. To compute traces we use

$$
\begin{aligned}
K\left(f_{*} X, f_{*} Y\right)= & \sum_{\imath=1}^{2 n+1} R^{A}\left(f_{*} E_{\imath}, f_{*} X ; f_{*} Y, f_{*} E_{\imath}\right) \\
& \left.+\sum_{a=1}^{k} R^{A}\left(\xi_{a}, f_{*} X ; f_{*} Y, \xi_{a}\right)+R^{A}\left(J_{A} \xi_{a}, f_{*} X ; f_{*} Y, J_{A} \xi_{a}\right)\right\} .
\end{aligned}
$$

We may assume that $E_{\alpha+n}=J E_{\alpha}, 1 \leqq \alpha \leqq n$. Consequently

$$
\sum_{\imath=1}^{2 n+1} \alpha(f)\left(E_{\imath}, E_{\imath}\right)=0 .
$$

Here $\alpha(f)$ is not the second fundamental form of $f$ (with respect to the Webster metrics of $M$ and $A$ ) but rather its pseudohermitian analogue. Nevertheless (as observed in the introduction) the 'true' second fundamental form of $f$ is traceless as well (and $f$ is a minimal isometric immersion). This is natural since pseudohermitian immersions appear to behave very much like holomorphic isometric immersions between Kaehlerian manifolds. The implications of minimality have been discussed in [D1] (cf. Theorems 7, 8 and 12 there). Next (37) leads to

$$
\begin{aligned}
\operatorname{Ric}(X, Y)= & K\left(f_{*} X, f_{*} Y\right) \\
& -\sum_{a=1}^{k}\left\{R^{A}\left(\xi_{a}, f_{*} X ; f_{*} Y, \xi_{a}\right)+R^{A}\left(J_{A} \xi_{a}, f_{*} X ; f_{*} Y, J_{A} \xi_{a}\right)\right\} \\
& -\sum_{\imath=1}^{2 n+1} g_{\Theta}\left(\alpha(f)\left(X, E_{\imath}\right), \alpha(f)\left(E_{\imath}, Y\right)\right),
\end{aligned}
$$


PSEUDOHERMITIAN IMMERSIONS, PSEUDO-EINSTEIN STRUCTURES, LEE CLASS 73 for any $X, Y \in T(M)$.

\section{Proof of Theorem 2}

We shall need the $1^{\text {st }}$ Bianchi identity for $\nabla^{A}$ (cf. e.g. S. Kobayashi \& K. Nomizu [KN], vol. I, p. 135)

$$
\sum_{V Z W} R^{A}(V, Z) W=\sum_{V Z W}\left\{\left(\nabla_{V}^{A} \operatorname{Tor}_{A}\right)(Z, W)+\operatorname{Tor}_{A}\left(\operatorname{Tor}_{A}(V, Z), W\right)\right\},
$$

for any $V, Z, W \in T(A)$. Here $\sum_{V Z W}$ denotes the cyclic sum over $V, Z, W$. Set $V=f_{*} X, Z=J_{A} f_{*} Y$ and $W=\xi_{a}$ in (39) and take the inner product of the resulting identity with $J_{A} \xi_{a}$. This procedure leads to

$$
\begin{aligned}
& R^{A}\left(f_{*} X, J_{A} f_{*} Y ; \xi a, J_{A} \xi a\right) \\
= & R^{A}\left(\xi_{a}, J_{A} f_{*} Y ; f_{*} X, J_{A} \xi_{a}\right)-R^{A}\left(\xi_{a}, f_{*} X ; J_{A} f_{*} Y, J_{A} \xi_{a}\right)+E_{a}(X, Y),
\end{aligned}
$$

where

Note that

$$
\begin{aligned}
E_{a}(X, Y)= & g_{\Theta}\left(\left(\nabla_{f_{*} X}^{A} \operatorname{Tor}_{A}\right)\left(J_{A} f_{*} Y, \xi_{a}\right), J_{A} \xi_{a}\right) \\
& +g_{\Theta}\left(\left(\nabla_{J_{A}}^{A} \operatorname{Tor}_{A}\right)\left(\xi_{a}, f_{*} X\right), J_{A} \xi_{a}\right) \\
& +g_{\theta}\left(\left(\nabla_{\xi_{a}}^{A} \operatorname{Tor}_{A}\right)\left(f_{*} X, J_{A} f_{*} Y\right), J_{A} \xi_{a}\right) \\
& +g_{\Theta}\left(\operatorname{Tor}_{A}\left(\operatorname{Tor}_{A}\left(f_{*} X, J_{A} f_{*} Y\right), \xi_{a}\right), J_{A} \xi_{a}\right) \\
& +g_{\theta}\left(\operatorname{Tor}_{A}\left(\operatorname{Tor}_{A}\left(J_{A} f_{*} Y, \xi_{a}\right), f_{*} X\right), J_{A} \xi_{a}\right) \\
& +g_{\Theta}\left(\operatorname{Tor}_{A}\left(\operatorname{Tor}_{A}\left(\xi_{a}, f_{*} X\right), J_{A} f_{*} Y\right), J_{A} \xi_{a}\right)
\end{aligned}
$$

$$
R^{A}(V, Z) J_{A} W=J_{A} R^{A}(V, Z) W,
$$

(as a consequence of $\nabla^{A} J_{A}=0$ ) for any $V, Z, W \in T(A)$. By (41) and $\Theta\left(\xi_{a}\right)=0$ we obtain

$$
R^{A}\left(\xi_{a}, f_{*} X ; J_{A} f_{*} Y, J_{A} \xi_{a}\right)=R^{A}\left(\xi_{a}, f_{*} X ; f_{*} Y, \xi_{a}\right) .
$$

Next, replace $\xi$ by $\xi_{a}$ and $Y$ by $J Y$ in (36) so that to obtain (provided $R^{\perp}=0$ )

$$
\begin{aligned}
& R^{A}\left(f_{*} X, f_{*} J Y ; \xi_{a}, J_{A} \xi_{a}\right) \\
= & 2 g_{\theta}\left(a_{\xi_{a}}^{2} X, Y\right)-2 g_{\theta}\left(\operatorname{Tor}_{A}\left(f_{*} a_{\xi_{a}} X, f_{*} J Y\right), J_{A} \xi_{a}\right),
\end{aligned}
$$

for any $X, Y \in T(M)$. At this point we may use (42)-(43) such that to write (40) as follows

$$
\begin{aligned}
& 2 g_{\theta}\left(a_{\xi_{a}}^{2} X, Y\right)-2 g_{\theta}\left(\operatorname{Tor}\left(f_{*} a_{\xi_{a}} X, f_{*} J Y\right), J_{A} \xi_{a}\right) \\
= & R^{A}\left(\xi_{a}, J_{A} f_{*} Y ; f_{*} X, J_{A} \xi_{a}\right)-R^{A}\left(\xi_{a}, f_{*} X ; f_{*} Y, \xi_{a}\right)+E_{a}(X, Y),
\end{aligned}
$$

for any $X, Y \in T(M)$. To deal with the torsion terms in (44) we need the following 
LEMMA 2. Let $T_{a}=1 / 2\left(E_{\alpha}-i J E_{\alpha}\right), 1 \leqq \alpha \leqq n$. Then

$$
E_{a}\left(T_{\alpha}, T_{\bar{\beta}}\right)=i g_{\theta}\left(\tau_{A} \xi_{a}, J_{A} \xi_{a}\right) h_{\alpha \bar{\beta}} .
$$

The proof of Lemma 2 is a straightforward consequence of

$$
\begin{aligned}
& \operatorname{Tor}_{A}(Z, W)=\operatorname{Tor}_{A}(\bar{Z}, \bar{W})=0, \\
& \operatorname{Tor}_{A}(Z, \bar{W})=i G_{\boldsymbol{\theta}}(Z, \bar{W}) T_{A}, \\
& \tau_{A} Z \in T_{0,1}(A),
\end{aligned}
$$

for any $Z, W \in T_{1,0}(A)$.

LEMMA 3. For any $X, Y Z, W \in H(A)$ the following identity holds

$$
\begin{aligned}
& R^{A}(X, Y ; Z, W) \\
= & R^{A}(Z, W ; X, Y)+A_{\Theta}(Y, Z) \Omega_{\theta}(W, X)+A_{\Theta}(X, W) \Omega_{\theta}(Z, Y) \\
& +A_{\Theta}(W, Y) \Omega_{\theta}(X, Z)+A_{\Theta}(Z, X) \Omega_{\Theta}(Y, W),
\end{aligned}
$$

where $A_{\Theta}(X, Y)=g_{\theta}\left(\tau_{A} X, Y\right)$.

We shall prove Lemma 3 later on. Using (46) we may compute the first curvature term in (44) as

$$
\begin{aligned}
& R^{A}\left(\xi_{a}, f_{*} J Y ; f_{*} X, J_{A} \xi_{a}\right) \\
= & R^{A}\left(f_{*} X, J_{A} \xi_{a} ; \xi_{a}, f_{*} J Y\right)+A_{\Theta}\left(f_{*} J Y, f_{*} X\right) \Omega_{\Theta}\left(J_{A} \xi_{a}, \xi_{a}\right) \\
& +A_{\Theta}\left(\xi_{a}, J_{A} \xi_{a}\right) \Omega_{\Theta}\left(f_{*} X, f_{*} J Y\right),
\end{aligned}
$$

for any $X, Y \in H(M)$. Also

$$
R^{A}\left(f_{*} X, J_{A} \xi_{a} ; \xi_{a}, f_{*} J Y\right)=-R^{A}\left(J_{A} \xi_{a}, f_{*} X ; f_{*} Y, J_{A} \xi_{a}\right) .
$$

Let us substitute from (47)-(48) into (44) and use the identities

so that to yield

$$
\begin{aligned}
& A_{\Theta}\left(f_{*} X, f_{*} Y\right)=A(X, Y), \\
& \Omega_{\Theta}\left(f_{*} X, f_{*} J Y\right)=-g_{\Theta}(X, Y),
\end{aligned}
$$

$$
\begin{aligned}
& 2 g_{\theta}\left(a_{\xi_{a}}^{2} X, Y\right)-2 g_{\theta}\left(\operatorname{Tor}_{A}\left(f_{*} a_{\xi_{a}} X, f_{*} J Y\right), J_{A} \xi_{a}\right) \\
= & -R^{A}\left(J_{A} \xi_{a}, f_{*} X ; f_{*} Y, J_{A} \xi_{a}\right)-R^{A}\left(\xi_{a}, f_{*} X ; f_{*} Y, \xi_{a}\right) \\
& +A(X, J Y)-g_{\theta}(X, Y) A_{\theta}\left(\xi_{a}, J_{A} \xi_{a}\right)+E_{a}(X, Y),
\end{aligned}
$$

for any $X, Y \in H(M)$. On the other hand (using (27)) one may show that 


$$
\begin{aligned}
& \sum_{\imath=1}^{2 n+1} g_{\theta}\left(\alpha(f)\left(X, E_{\imath}\right), \alpha(f)\left(E_{\imath}, Y\right)\right) \\
= & \sum_{a=1}^{k}\left\{2 g_{\theta}\left(a_{\xi_{a}}^{2} X, Y\right)+g_{\theta}\left(\operatorname{Tor}_{A}\left(f_{*} Y, f_{*} a_{\xi_{a}} X\right), \xi_{a}\right)\right. \\
& \left.-g_{\theta}\left(\operatorname{Tor}_{A}\left(f_{*} Y, f_{*} J a_{\xi_{a}} X\right), J_{A} \xi_{a}\right)\right\} .
\end{aligned}
$$

Finally, substitution from (49)-(50) into (38) gives

$$
\begin{aligned}
\operatorname{Ric}(X, Y)= & K\left(f_{*} X, f_{*} Y\right)-\sum_{a=1}^{k}\left\{g_{\Theta}\left(\operatorname{Tor}_{A}\left(f_{*} J a_{\xi_{a}} X, f_{*} Y\right), J_{A} \xi_{a}\right)\right. \\
& -g_{\Theta}\left(\operatorname{Tor}_{A}\left(f_{*} a_{\xi_{a}} X, f_{*} Y\right), \xi_{a}\right)-2 g_{\theta}\left(\operatorname{Tor}_{A}\left(f_{*} a_{\xi_{a}}, f_{*} J Y\right), J_{A} \xi_{a}\right) \\
& \left.-A(X, J Y)+g(X, Y) A_{\Theta}\left(\xi_{a}, J_{A} \xi_{a}\right)-E_{a}(X, Y)\right\}
\end{aligned}
$$

for any $X, Y \in H(M)$. Let us extend both sides of (51) by $C$-linearity to $H(M) \otimes C$. It follows that (51) holds for any $X, Y \in H(M) \otimes C$ (as both sides are $\mathcal{E} \otimes C$-linear and coincide on real vectors). Set $X=Z, Y=\bar{W}$, with $Z, W \in$ $T_{1,0}(M)$. We obtain

$$
\operatorname{Ric}(Z, \bar{W})=K\left(f_{*} Z, f_{*} \bar{W}\right)+\sum_{a=1}^{k}\left\{A_{\Theta}\left(\xi_{a}, J_{A} \xi_{a}\right) g_{\theta}(Z, \bar{W})-E_{a}(Z, \bar{W})\right\} .
$$

Finally, we set $Z=T_{\alpha}$ and $W=T_{\bar{\beta}}$ in (52) and use (45) of Lemma 2 so that to yield (26).

Q.E.D.

\section{Pseudohermitian Ricci curvature and the first Chern class of the normal bundle}

Let $(M, \theta)$ and $(A, \Theta)$ be two strictly pseudoconvex CR manifolds and $f: M \rightarrow A$ a pseudohermitian immersion. The purpose of the present section is the converse of Theorem 1, i.e. it may be asked whether (26) yields $R^{\perp}=0$. We establish the following weaker result. Let $\nu^{2 k}(f) \rightarrow M$ be the normal bundle of $f$. By a result in $[\mathrm{D} 1], \nu^{2 k}(f)_{x} \subset H(A)_{f(x)}$ for any $x \in M$ so that $J_{A}$ descends to a complex structure $J^{\perp}$ in $\nu^{2 k}(f)$. Extend $J^{\perp}$ by complex linearity to $\nu^{2 k}(f) \otimes C$ and let $\nu^{2 k}(f)^{1,0}$ be the eigenbundle corresponding to the eigenvalue $\imath$. We may state

THEOREM 3. Let $f: M \rightarrow A$ be a pseudohermitian immersion with the property $R_{\alpha \bar{\beta}}=K_{\alpha \bar{\beta}}$, where $K_{\alpha \bar{\beta}}=K\left(f_{*} T_{\alpha}, f_{*} T_{\bar{\beta}}\right)$. If the Tanaka-Webster connection of $A$ has parallel pseudohermitian torsion $\left(\nabla^{A} \tau_{A}=0\right)$ then

$$
c_{1}\left(\nu^{2 k}(f)^{1,0}\right)=0 \text {. }
$$

Throughout, if $E \rightarrow M$ is a $\boldsymbol{C}$-vector bundle then $c_{1}(E) \in H^{2}(M ; \boldsymbol{R})$ denotes its first Chern class. To prove Theorem 3 we need the following 
LEMMA 4. Let $f: M \rightarrow A$ be a pseudohermitian immersion. If the ambient space $A$ has parallel pseudohermitian torsion then

$$
\left(\nabla_{X} A\right)(Y, Z)=g_{\theta}(\alpha(f)(X, Z),(Q f) Z)+g_{\theta}((Q f) Y, \alpha(f)(X, Z)),
$$

for any $X, Y, Z \in T(M)$.

The proof of Lemma 4 follows from $\nabla^{A} \tau_{A}=0$ and (14)-(15), (17) in a straightforward manner. Recall that $c_{1}\left(T_{1,0} M\right)$ is represented by $(i / 2 \pi) d \omega_{\alpha}^{\alpha}$ where

and

$$
d \omega_{\alpha}^{\alpha}=R_{\alpha \bar{\beta}} \theta^{\alpha} \wedge \theta^{\bar{\beta}}+W_{\alpha \beta}^{\alpha} \theta^{\beta} \wedge \theta-W_{\bar{\alpha} \bar{\beta}}^{\bar{\alpha}} \theta^{\bar{\beta}} \wedge \theta
$$

where

$$
W_{\beta \gamma}^{\alpha}=A_{\beta \gamma, \bar{\sigma}} h^{\alpha \bar{\sigma}},
$$

$$
A_{\alpha \beta, \bar{\gamma}}=\left(\nabla_{T_{\bar{\gamma}}} A\right)\left(T_{\alpha}, T_{\beta}\right)
$$

are the covariant derivatives of the pseudohermitian torsion (with respect to the Tanaka-Webster connection). Also $\omega_{\beta}^{\alpha}$ are the connection 1-forms of $\nabla$. Cf. [L1], p. 162. Let $\left\{\Theta^{1}, \cdots, \Theta^{N}\right\}$ be the admissible coframe dual to $\left\{T_{1}, \cdots, T_{n}\right.$, $\left.\zeta_{1}, \cdots, \zeta_{k}\right\}$ where $\zeta_{a}=1 / 2\left(\xi_{a}-i J_{A} \xi_{a}\right)$. Then $f^{*} \Theta^{\alpha}=\theta^{\alpha}$ and $f^{*} \Theta^{a+n}=0$. Next $c_{1}$ $\left(T_{1,0} A\right)$ is represented by $(i / 2 \pi) d \Omega_{j}^{\jmath}$ where $\Omega_{j}^{\imath}$ are the connection 1 -forms of $\nabla^{A}$ and $\left(A_{\theta}\right)_{i j, \bar{k}}=0$ yields

$$
d \Omega_{j}^{j}=K_{j \bar{k}} \Theta^{\jmath} \wedge \Theta^{\bar{k}} .
$$

Finally (53) gives $A_{\alpha \beta, \bar{\gamma}}=0$ so that $f^{*} c_{1}\left(T_{1,0} A\right)=c_{1}\left(T_{1,0} M\right)$ and the direct sum decomposition

$$
T_{1,0}(A)_{f(x)}=\left[\left(d_{x} f\right) T_{1,0}(M)_{x}\right] \oplus \nu^{2 k}(f)_{x}^{1,0},
$$

for each $x \in M$, yields $c_{1}\left(\nu^{2 k}(f)^{1,0}\right)=0$.

Q.E.D.

Let $f: M \rightarrow A$ be a pseudohermitian immersion. Assume that $R^{A}=0$ (e.g. $A=H_{N}$ ). Then (38) gives

$$
\operatorname{Ric}(X, Y)=-\sum_{\imath=1}^{2 n+1} g_{\theta}\left(\alpha(f)\left(X, E_{\imath}\right), \alpha(f)\left(E_{\imath}, Y\right)\right),
$$

or (by computing traces)

$$
2 R=-\|\alpha(f)\|^{2} \leqq 0
$$

THEOREM 4. There is no pseudohermitian immersion of

$$
\left(H_{n}(s),|x|^{-2}\left\{d t+2 \sum_{\alpha=1}^{n}\left(x^{\alpha} d y^{\alpha}-y^{\alpha} d x^{\alpha}\right)\right\}\right)
$$

into a Tanaka-Webster flat strictly pseudoconvex CR manifold.

Proof. By a result of [D2], p. 42, we have 


$$
R_{\alpha \bar{\beta}}=(n+1)|x|^{-2}|z|^{2} h_{\alpha \bar{\beta}},
$$

or (by computing traces)

$$
R=n(n+1)|x|^{-2}|z|^{2} .
$$

Assume there is strictly pseudoconvex $\mathrm{CR}$ manifold $A$ with $R^{A}=0$ and a pseudohermitian immersion $f: H_{n}(s) \rightarrow A$. Then (56) contradicts (54) and Theorem 4 is completely proved.

We end this section with a remark regarding the analogy with Kählerian geometry (cf. [CL], p. 554). Let $f: M \rightarrow A$ be a pseudohermitian immersion. Assume that $c_{1}\left(T_{1,0}(M)\right)=0$. Then, there is a real 1-form $\eta$ on $M$ so that

$$
\Gamma=d \eta,
$$

where $\Gamma=(i / 2 \pi) d \omega_{\alpha}^{\alpha}$. A $C$-valued 2 -form $\eta$ on $M$ is a $(1,1)$-form if $\left.T\right\lrcorner \eta=0$ and $\eta(Z, W)=\eta(\bar{Z}, \bar{W})=0$ for any $Z, W \in T_{1,0}(M)$. Let $\Lambda^{1,1}(M)$ be the bundle of $(1,1)$-forms on $M$. Define $L_{\theta}: \mathcal{E}(M) \otimes C \rightarrow \Lambda^{1,1}(M)$ by setting $L_{\theta} f=f \Omega_{\theta}$ for any $C^{\infty}$ function $f: M \rightarrow C$. Next we need $\Lambda_{\theta}: \Lambda^{1,1}(M) \rightarrow \mathcal{E}(M) \otimes C$ given by $\left(\Lambda_{\theta} \Psi, f\right)_{\theta}=\left(\Psi, L_{\theta} f\right)_{\theta}$ for any $\Psi \in \Gamma^{\infty}\left(\Lambda^{1,1}(M)\right)$. Here $(,)_{\theta}$ is the usual $L^{2}$ inner product on $(M, \theta)$, i.e.

$$
(\phi, \phi)_{\theta}=\int_{M}\langle\phi, \phi\rangle \theta \wedge(d \theta)^{n},
$$

for any $(1,1)$-forms $\phi, \phi$ on $M$ (at least one of compact support) where $\langle\phi, \phi\rangle$ $=\phi_{\alpha \bar{\beta}} \psi^{\alpha \bar{\beta}}$ and $\phi=\phi_{\alpha \bar{\beta}} \theta^{\alpha} \wedge \theta^{\bar{\beta}}, \quad \phi=\phi_{\alpha \bar{\beta}} \theta^{\alpha} \wedge \theta^{\bar{\beta}}$, and $\phi^{\alpha \bar{\beta}}=\overline{\phi^{\bar{\alpha} \beta}}, \quad \phi^{\bar{\alpha} \beta}=\phi_{\lambda \bar{\mu}} h^{\lambda \bar{\alpha}} h^{\bar{\mu} \beta}$. We may extend $\Lambda_{\theta}$ to an operator $\Lambda_{\theta}: \Lambda^{2} T * M \otimes C \rightarrow \mathcal{E}(M) \otimes C$ by declaring it to be zero on $\Lambda^{0,2} \oplus \Lambda^{2,0}$ (a $(2,0)$-form $\eta$ is a $C$-valued 2 -form satisfying $\left.\left.T_{0,1}(M)\right\lrcorner \eta=0\right)$. Then

$$
\Lambda_{\theta} \Gamma=-\frac{1}{\pi} R
$$

and we may apply $\Lambda_{\theta}$ to (57) so that to yield

$$
\frac{1}{2 \pi} R=n \eta_{0}+i \operatorname{div}(Z)
$$

where $\eta=\eta_{\alpha} \theta^{\alpha}+\eta_{\bar{\alpha}} \theta^{\bar{\alpha}}+\eta_{0} \theta$ and $Z=Z^{\bar{\alpha}} T_{\bar{\alpha}}-Z^{\alpha} T_{\alpha}$ with $Z^{\alpha}=h^{\alpha \bar{\beta}} \eta_{\bar{\beta}}$. The divergence in (58) is taken with respect to the volume form $\phi=\theta \wedge(d \theta)^{n}$ (i.e. $\operatorname{div}(Z) \psi=\mathcal{L}_{Z} \psi$, where $\mathcal{L}$ denotes the Lie derivative). Therefore, if $\int_{M} \eta_{0} \psi \geqq 0$ then (54) gives $\alpha(f)=0$ and thus $R^{\perp}=0$ (as a consequence of (20)) provided that $R^{A}=0$. Yet, by a result of [L1], p. 169, if $(M, \theta)$ is pseudo-Einstein, one representative of $\Gamma$ is $\eta=(1 / 2 \pi n) R \theta$ so that (in view of (54)) the hypothesis $\int_{M} \eta_{0} \phi \geqq 0$ is generically not satisfied. Indeed, let $\eta^{\prime}$ be any other real 1 -form so that $\Gamma=d \eta^{\prime}$. If for instance $H^{1}(M ; \boldsymbol{R})=0$ then $\eta^{\prime}=\eta+d u$ for some $C^{\infty}$ function $u: M \rightarrow \boldsymbol{R}$ and (58) yields $\int_{M} T(u) \phi=0$, that is $\int_{M} \eta_{0}^{\prime} \psi=\int_{M} \eta_{0} \phi \leqq 0$. 


\section{Curvature properties of the Tanaka-Webster connection}

The main purpose of this section is to prove Lemma 3. Let $(M, \theta)$ be a nondegenerate CR manifold. Let $R, R^{\theta}$ be the curvature tensor fields of $\nabla, \nabla^{\theta}$, respectively. Taking into account (9) we may derive the following identity

$$
\begin{aligned}
R^{\theta}(X, Y) Z= & R(X, Y) Z-(L X \wedge L Y) Z+\theta(Z) S(X, Y) \\
& -g_{\theta}(S(X, Y), Z) T+2 \theta(Z)(\theta \wedge \theta)(X, Y) \\
& -2 g_{\theta}((\theta \wedge \Theta)(X, Y), Z) T-\frac{1}{2} \Omega_{\theta}(X, Y) J Z,
\end{aligned}
$$

for any $X, Y, Z \in T(M)$. We need to explain the notations in (59). Firstly $L=\tau+(1 / 2) J$. Next $(X \wedge Y) Z=g_{\theta}(Y, Z) X-g_{\theta}(X, Z) Y$ for any $X, Y Z \in T(M)$. Also we set

$$
S(X, Y)=\left(\nabla_{X} \tau\right) Y-\left(\nabla_{Y} \tau\right) X
$$

Finally, the operator $\mathcal{O}$ is given by $\theta=\tau^{2}+J \tau-(1 / 4) I$, where $I$ denotes the identical transformation. The proof of (59) is a rather lengthy computation based on the identities

$$
\begin{aligned}
& \nabla J=0, \quad \theta \circ J=0, \quad \theta \circ \tau=0, \\
& \nabla_{X}^{\theta} T=L X, \quad \nabla \Omega_{\theta}=0, \quad \nabla \theta=0, \\
& A(X, J Y)=A(J X, Y), \\
& \Omega_{\theta}(X, \tau Y)+\Omega_{\theta}(\tau X, Y)=0, \\
& L^{*}=\tau-\frac{1}{2} J, \quad \tau L^{*}=L \tau, \\
& (d \theta)(X, Y)=-\frac{1}{2} \Omega_{\theta}(X, Y),
\end{aligned}
$$

for any $X, Y \in T(M)$ (and is left as an exercise to the reader).

Let $X, Y, Z, W \in H(M)$. Take the inner product of (59) with $W$. This procedure furnishes

$$
\begin{aligned}
R^{\theta}(X, Y ; Z, W)= & R(X, Y ; Z, W)-g_{\theta}((L X \wedge L Y) Z, W) \\
& +\frac{1}{2} \Omega_{\theta}(X, Y) \Omega_{\theta}(Z, W),
\end{aligned}
$$

for any $X, Y, Z, W \in H(M)$. Then we may use (61) twice so that to yield

$$
\begin{aligned}
R(X, Y ; Z, W)= & R(Z, W ; X, Y)+g_{\theta}((L X \wedge L Y) Z, W) \\
& -g_{\theta}((L Z \wedge L W) X, Y),
\end{aligned}
$$

which in turn leads to (46) of Lemma 3 . The general philosophy of this 
procedure is that one uses the known symmetries of the Riemann-Christoffel tensor $R_{j k l m}^{\theta}$ of $\left(M, g_{\theta}\right)$ via (59), rather than establishing similar properties for $R_{j k l m}$. Nevertheless, let us observe that $R_{j k l m}+R_{k j l m}=0$ because $R$ is a 2 -form, and $R_{j k l m}+R_{j k m l}=0$ because $\nabla g_{\theta}=0$. The missing property is obviously $R_{j k l m}=R_{l m j k}$. Any tentative to obtain a CR analogue of $R_{j k l m}^{\theta}=R_{l m j k}^{\theta}$ passing through the Bianchi identities (of the Tanaka-Webster connection) would have to deal with the torsion terms there. As remarked in section $2, R_{\alpha \bar{\beta}}$ is only a fragment of Ric and (as a consequence of (59)) we have

$$
\begin{aligned}
& R_{\alpha \bar{\beta}}^{\theta}=h_{\alpha \bar{\beta}}-\frac{1}{2} R_{\alpha \bar{\beta}}, \\
& R_{\alpha \beta}=i(n-1) A_{\alpha \beta}, \\
& R_{0 \beta}=S_{\bar{\alpha} \beta}^{\bar{\alpha}}, \quad R_{\alpha 0}=R_{00}=0 .
\end{aligned}
$$

Here $S_{\bar{\beta} \gamma}^{\bar{\alpha}}$ are (among) the complex components of $S$ (given by (60)). Also we set $R_{\alpha \bar{\beta}}^{\theta}=\operatorname{trace}\left\{X \mapsto R^{\theta}\left(X, T_{\alpha}\right) T_{\bar{\beta}}\right\}$. The proof of (62) is omitted. Finally, we wish to show that

$$
2 R=\operatorname{trace}(\mathrm{Ric}) \text {. }
$$

Note that (63) was employed to derive the identity (54). By (59) the following identities hold

$$
\begin{aligned}
& \operatorname{Ric}\left(E_{\alpha}, E_{\beta}\right)=i(n-1)\left(A_{\alpha \beta}-A_{\bar{\alpha} \bar{\beta}}\right)+R_{\alpha \bar{\beta}}+R_{\bar{\alpha} \beta}, \\
& \operatorname{Ric}\left(J E_{\alpha}, E_{\beta}\right)=-(n-1)\left(A_{\alpha \beta}+A_{\bar{\alpha} \bar{\beta}}\right)+i\left(R_{\alpha \bar{\beta}}-R_{\bar{\alpha} \beta}\right), \\
& \operatorname{Ric}\left(E_{\alpha}, J E_{\beta}\right)=-(n-1)\left(A_{\alpha \beta}+A_{\bar{\alpha} \bar{\beta}}\right)+i\left({ }_{\beta \bar{\alpha}}-R_{\bar{\beta} \alpha}\right), \\
& \operatorname{Ric}\left(J E_{\alpha}, J E_{\beta}\right)=-i(n-1)\left(A_{\alpha \beta}-A_{\bar{\alpha} \bar{\beta}}\right)+R_{\alpha \bar{\beta}}+R_{\bar{\alpha} \beta} .
\end{aligned}
$$

Then $\operatorname{trace}(\operatorname{Ric})=g^{\imath \jmath} \operatorname{Ric}\left(E_{\imath}, E_{\jmath}\right)$, where

$$
\begin{aligned}
& g^{\alpha+n, \beta+n}=g^{\alpha \beta}, \quad g^{\alpha, \beta+n}=-g^{\alpha+n, \beta}, \\
& g^{\alpha 0}=g^{0 \alpha}=0, \quad g^{00}=1, \\
& g^{\alpha \beta}=\frac{1}{4}\left(h^{\alpha \bar{\beta}}+h^{\bar{\alpha} \beta}\right), \quad g^{\alpha, \beta+n}=\frac{i}{4}\left(h^{\alpha \bar{\beta}}-h^{\bar{\alpha} \beta}\right),
\end{aligned}
$$

and the identities (64)-(67) lead to (63).

Q.E.D.

\section{Proof of Theorem 1}

Let $U=\left\{U_{\alpha}\right\}_{\alpha \in I} \in \operatorname{Cov}(M)$ and $u_{\beta \alpha} \in \mathscr{Q}\left(U_{\alpha} \cap U_{\beta}\right)$ so that $i_{\alpha \beta}^{*} \theta_{\beta}=\exp \left(2 u_{\beta \alpha}\right) i_{\beta \alpha}^{*} \theta_{\alpha}$ where $i_{\alpha \beta}: U_{\alpha} \cap U_{\beta} \rightarrow U_{\beta}$ are inclusions. Then $\gamma(M) \in H^{1}(M, \mathscr{P})$ is the equivalence class of $[c] \in H^{1}(N(\mathcal{U}), \mathscr{Q})$, where $c: \Delta(\alpha \beta) \mapsto u_{\beta \alpha}$. Let $\mathscr{W} \in \operatorname{Cov}(M)$ so that $\mathscr{W}<\mathcal{U}$, $\mathscr{W}<C V$. Set $\mathscr{W}=\left\{W_{\alpha}\right\}_{\alpha \in J}$. There are maps $\phi: J \rightarrow I$ and $\phi: J \rightarrow \Sigma$ so that 
$W_{a} \subset U_{\phi(a)} \cap V_{\psi(a)}$ for each $a \in J$. Set $\lambda_{a}=r_{a}^{*} \theta_{\phi(a)}$ and $\mu_{a}=s_{a}^{*} \varphi_{\psi(a)}$ where $r_{a}: W_{a} \rightarrow U_{\phi(a)}$ and $s_{a}: W_{a} \rightarrow V_{\psi(a)}$ are inclusions. Note that

$$
k_{a b}^{*} \lambda_{b}=\exp \left(2 h_{b a}\right) k_{b a}^{*} \lambda_{a},
$$

where $k_{a b}^{*}: W_{a} \cap W_{b} \rightarrow W_{b}$ are inclusions and $h_{b a}=u_{\beta \alpha^{\circ}} r_{a b}$ with $\alpha=\phi(a)$ and $\beta=\phi(b)$, and $r_{a b}: W_{a} \cap W_{b} \subset U_{\alpha} \cap U_{\beta}$. In other words $h_{b a}=\rho_{\phi \sigma, \sigma}\left(u_{\beta \alpha}\right)$ where $\rho_{\psi \sigma, \sigma}: \mathscr{L}(\cap \phi \sigma) \rightarrow \mathscr{Q}(\cap \sigma)$ is the restriction map $(\sigma=\Delta(a b) \in N(\mathscr{W}))$ and $\phi: N(\mathscr{W}) \rightarrow$ $N(\mathcal{U})$ the natural simplicial map. If $\tilde{\phi}: \mathcal{C}^{1}(N(\mathcal{U}), \mathscr{P}) \rightarrow \mathcal{C}^{1}(N(\mathscr{W}), \mathscr{P})$ is the induced map on cochains, then $(\tilde{\phi} c) \sigma=h_{b a}$, and if $\phi^{*}: H^{1}(N(\mathcal{U}), \mathscr{Q}) \rightarrow H^{1}(N(\mathscr{W}), \mathscr{Q})$ is the induced map on cohomology then $\phi^{*} g=[\tilde{\phi} c]$ with $g=[c]$ so that

$$
\gamma(M)=\left[\phi^{*} g\right],
$$

(one checks that $g \sim \phi^{*} g$ by looking at $\mathscr{W}$ as a common refinement of itself and $U)$. Both $\left(W_{a}, \lambda_{a}\right)$ and $\left(W_{a}, \mu_{a}\right)$ are strictly pseudoconvex $\mathrm{CR}$ manifolds so that

$$
\mu_{a}=\exp \left(2 v_{a}\right) \lambda_{a}
$$

for some $v_{a} \in \mathcal{E}\left(W_{a}\right)$. Let $v \in \mathcal{C}^{1}(N(\mathscr{W}), \mathcal{E})$ be given by $v: \Delta(a) \mapsto v_{a}$. Similar to (68) we have

$$
k_{a b}^{*} \mu_{b}=\exp \left(2 \tilde{h}_{b a}\right) k_{b a}^{*} \mu_{a},
$$

where $\tilde{h}_{b a}=\tilde{u}_{j i}{ }^{\circ} s_{a b}$ with $i=\phi(a), \jmath=\phi(b)$ and $s_{a b}: W_{a} \cap W_{b} \subset V_{i} \cap V_{\jmath}$. Also $\tilde{u}_{j i}=$ $U_{j i} \circ f_{\imath \jmath}$ and $f_{\imath \jmath}: V_{i} \cap V_{j} \rightarrow D_{i} \cap D$, is induced by $f$. Finally (68)-(70) lead to

$$
\tilde{h}_{b a}=v_{b} \circ k_{a b}+h_{b a}-v_{a} \circ k_{b a} .
$$

Let $\jmath: \mathcal{C}^{1}(N(\mathscr{W}), \mathscr{Q}) \rightarrow \mathcal{C}^{1}(N(\mathscr{W}), \mathcal{E})$ be induced by the natural sheaf morphism $\mathscr{Q} \rightarrow \mathcal{E}$ (i.e. $\mathscr{Q}(U) \rightarrow \mathcal{E}(U)$ is the inclusion, for each $U \subseteq M$ open). Then (71) may be written

where

$$
j \tilde{\phi} f * C=\boldsymbol{\delta}_{\varepsilon} v+\jmath \tilde{\phi} c
$$

$$
\delta_{\mathcal{E}}: \mathcal{C}^{1}(N(\mathscr{W}), \mathcal{E}) \rightarrow \mathcal{C}^{2}(N(\mathscr{W}), \mathcal{E}),
$$

is the coboundary operator. Consequently

$$
j \phi_{\mathcal{V W}} f^{*} G=j \phi_{\mathcal{q} w} g,
$$

where $j: H^{1}(N(\mathscr{W}), \mathscr{P}) \rightarrow H^{1}(N(\mathscr{W}), \mathcal{E})$. Finally, as $j$ and $\phi^{*}$ (respectively $j$ and $\left.\phi^{*}\right)$ commute it follows that $j\left(f^{*} \gamma(A)-\gamma(M)\right)=0$. Note that in general $\operatorname{Ker}(j) \neq 0$ (because $B^{1}(N(\mathscr{W}), \mathscr{P}) \subset B^{1}(N(\mathscr{W}), \mathcal{E})$, strict inclusion). If each $\mu_{a}$ is pseudoEinstein then $v_{a} \in \mathscr{P}\left(W_{a}\right)$ and (71) may be written

$$
\tilde{\phi} f^{*} C=\delta v+\tilde{\phi} c
$$

where $\delta: \mathcal{C}^{1}(N(\mathscr{W}), \mathscr{P}) \rightarrow \mathcal{C}^{2}(N(\mathscr{W}), \mathscr{Q})$ is the coboundary operator. Thus 
PSEUDOHERMITIAN IMMERSIONS, PSEUDO-EINSTEIN STRUCTURES, LEE CLASS 81

that is

$$
\phi_{\curlyvee \mathfrak{W}} f * G=\phi_{\cup \mathscr{W}} g,
$$

$$
f * r(A)=\gamma(M),
$$

and Theorem 1 is completely proved.

\section{Examples}

1) (Heisenberg groups)

Let $H_{n}$ be the Heisenberg group endowed with the (strictly pseudoconvex) $\mathrm{CR}$ structure spanned by

$$
T_{\alpha}=\frac{\partial}{\partial z_{\alpha}}+i \bar{z}_{\alpha} \frac{\partial}{\partial t},
$$

(the Lewy operators). Fix the contact 1 -form $\theta_{0}$ on $H_{n}$ given by

$$
\theta_{0}=d t+i \sum_{\alpha=1}^{n}\left\{z_{\alpha} d \bar{z}_{\alpha}-\bar{z}_{\alpha} d z_{\alpha}\right\} .
$$

The map $f: H_{n} \rightarrow H_{N}, N=n+k, k \geqq 1$, induced by the natural inclusion $\boldsymbol{C}^{n} \rightarrow \boldsymbol{C}^{N}$ (i.e. $\left.f(z, t)=(z, 0, t), 0 \in C^{k}\right)$ is a pseudohermitian immersion with a flat normal Tanaka-Webster connection. Indeed, let $(w, s)=\left(w_{1}, \cdots, w_{N}, s\right)$ be the natural coordinates on $H_{N}$. Then

$$
W_{\jmath}=\frac{\partial}{\partial w_{j}}+i \bar{w}_{j} \frac{\partial}{\partial s}
$$

span the CR structure of $H_{N}$ while

$$
\Theta_{0}=d s+\sum_{j=1}^{N}\left\{w_{j} d \bar{w}_{j}-\bar{w}_{j} d w_{j}\right\}
$$

is a contact 1-form on $H_{N}$ (whose corresponding Levi form is positive definite). Next $f_{*} T_{\alpha}=W_{\alpha}, f^{*} \Theta_{0}=\theta_{0}$ and $\operatorname{nor}\left(T_{A}\right)=0$ (here $A=H_{N}, T_{A}=\partial / \partial s$ ) by straightforward calculation. Finally $R^{\perp}=0$ as a consequence of (20) (the $\mathrm{CR}$ analogue of the Ricci equation) for $A=H_{N}$.

2) (Quotients of Heisenberg groups by discrete groups of dilations)

Let $H_{n}(s), 0<s<1$, carry the $\mathrm{CR}$ structure induced by the covering map $\pi: H_{n}-\{0\} \rightarrow H_{n}(s)$, and the contact 1-form $\theta$ given by

$$
\theta_{\pi(x)}=|x|^{-2} \theta_{0, x^{\circ}}\left(d_{x} \pi\right)^{-1},
$$

for any $x \in H_{n}-\{0\}$. The map $F: H_{n}(s) \rightarrow H_{N}(s)$ induced by $f: H_{n}-\{0\} \rightarrow H_{N}-\{0\}$ (i.e. $F \circ \pi=\Pi \circ f$, where $\Pi: H_{N}-\{0\} \rightarrow H_{N}(s)$ is the natural covering map) is a pseudohermitian immersion. Indeed, if $H_{N}(s)$ is endowed with the contact 1-form $\Theta$ given by

$$
\Theta_{I(X)}=|X|^{-2} \Theta_{0, X} \circ\left(d_{X} \Pi\right)^{-1},
$$


for any $X \in H_{N}-\{0\}$ then $|f(x)|=|x|, x \in H_{n}$, yields $F^{*} \Theta=\theta$ (i.e. $F$ is isopseudohermitian). Moreover, we may write (72)-(73) as

$$
\theta=e^{2 u} \theta_{0}, \quad \Theta=e^{2 U} \Theta_{0},
$$

(with $U=\log |X|^{-1}$ and $u=U \circ f$ ). Therefore, the characteristic directions $T$ and $T_{A}$ of $\left(H_{n}(s), \theta\right)$ and $\left(H_{N}(s), \Theta\right)$ are respectively given by

$$
\begin{aligned}
& T=e^{-2 u}\left\{\frac{\partial}{\partial t}-2 i u^{\beta} T_{\beta}+2 i u^{\bar{\beta}} T_{\bar{\beta}}\right\}, \\
& T_{A}=e^{-2 U}\left\{\frac{\partial}{\partial s}-2 i U^{j} W_{j}+2 i U^{j} W_{\bar{j}}\right\} .
\end{aligned}
$$

Note that

and

$$
U^{j}(f(x))=|x|^{2} W_{j}(U)_{f(x)}
$$

$$
W_{j}(U)=-\frac{1}{2}|X|^{-4} w_{j} \bar{\Phi},
$$

where $\Phi(w, s)=|w|^{2}+i s$ (note that $\bar{\Phi}$ is CR-holomorphic). Finally

and

$$
\begin{aligned}
& U^{\alpha}=|x|^{2} U_{\bar{\alpha}}, \\
& U_{\bar{\alpha}} \circ f=u_{\bar{\alpha}}, \quad U^{\alpha} \circ f=u^{\alpha},
\end{aligned}
$$

$$
T_{\bar{\alpha}}(u)=-\frac{1}{2}|x|^{-4} z_{\alpha} \bar{\phi},
$$

(where $\phi=\Phi \circ f$ ) yield $f_{*} T=T_{A}$. Next, let us compute the curvature of the normal Tanaka-Webster connection $\nabla^{\perp}$ of $F$. We perform our task in a more general setting, as follows. Let $f: M \rightarrow A$ be a pseudohermitian immersion between $(M, \theta)$ and $(A, \Theta)$ and set $\hat{\theta}=e^{2 u} \theta, \hat{\Theta}=e^{2 U} \Theta$ with $U \in C^{\infty}(A), u=U \circ f$. Readily $f * \hat{\Theta}=\hat{\theta}$. Set

$$
\hat{T}=e^{-2 u}\left\{T-2 i u^{\beta} T_{\beta}+2 i u^{\bar{\beta}} T_{\bar{\beta}}\right\},
$$

where $T$ is the characteristic direction of $(M, \theta)$. As $U^{\alpha} \circ f=u^{\alpha}$ we obtain

$$
\begin{aligned}
& \hat{T}_{A}(f(x))=\left(f_{*} \hat{T}\right)(f(x)) \\
& \quad+2 i e^{-2 u(x)}\left\{U^{\overline{a+n}}(f(x)) W_{\overline{a+n}}(f(x))-U^{a+n}(f(x)) W_{a+n}(f(x))\right\},
\end{aligned}
$$

for any $x \in M$. Thus $f_{*} \hat{T}=\hat{T}_{A}$ (i.e. $f$ is a pseudohermitian immersion from $(M, \hat{\theta})$ into $(A, \hat{\Theta}))$ if and only if $\zeta_{\bar{\alpha}}(U)=0$. Let us look now at the relation between $\nabla^{\perp}$ and $\hat{\nabla}^{\perp}$ (the normal Tanaka-Webster connection of $(M, \hat{\theta})$ in $(A, \hat{\Theta})$ ). Let $\hat{\nu}^{2 k}(f)_{x}$ be the orthogonal complement (with respect to $\left.g_{\hat{\theta}, f(x)}\right)$ of $\left(d_{x} f\right) T_{x}(M)$ in $T_{f(x)}(A)$, for any $x \in M$. Then $\hat{\nu}^{2 k}(f)_{x}=\nu^{2 k}(f)_{x}$, although the Webster metrics $g_{\hat{\theta}}, g_{\boldsymbol{\theta}}$ are not conformally related.

Assume from now on that $f$ is a pseudohermitian immersion both as a map of $(M, \theta)$ into $(A, \Theta)$, respectively of $(M, \hat{\theta})$ into $(A, \hat{\Theta})$. We need to recall 
PSEUDOHERMITIAN IMMERSIONS, PSEUDO-EINSTEIN STRUCTURES, LEE CLASS 83

LEMMA 5 (cf. [D2], p. 39). Let $\left(M, T_{1,0}(M), \theta, T\right)$ be a non-degenerate $C R$ manifold. Then, under a transformation $\hat{\theta}=e^{2 u} \theta$, the Christoffel symbols of the Tanaka-Webster connection of $\left(T_{1,0}(M), \theta\right)$ and $\left(T_{1,0}(M), \hat{\theta}\right)$ are related by

$$
\begin{aligned}
& \hat{\Gamma}_{\beta \alpha}^{\sigma}=\Gamma_{\beta \alpha}^{\sigma}+2 u_{\beta} \delta_{\alpha}^{\sigma}+2 u_{\alpha} \delta_{\beta}^{\sigma}, \\
& \hat{\Gamma}_{\bar{\beta} \alpha}^{\sigma}=\Gamma_{\bar{\beta} \alpha}^{\sigma}-2 u^{\sigma} h_{\bar{\beta} a}, \\
& e^{2 u} \hat{\Gamma}_{\hat{0} \alpha}^{\sigma}=\Gamma_{0 \alpha}^{\sigma}+2 u_{0} \delta_{\alpha}^{\sigma}+i u_{\alpha},{ }^{\sigma}+2 i \Gamma_{\bar{\mu} \alpha}^{\sigma} u^{\bar{\mu}}-2 i \Gamma_{\mu \alpha}^{\sigma} u^{\mu},
\end{aligned}
$$

where $u_{\alpha}{ }^{\sigma}=u_{\alpha, \bar{\beta}} h^{\sigma \bar{\beta}}$.

Using Lemma 5, the identity (15) and

$$
\hat{\nabla}_{f_{*} X}^{A} \xi=-f_{*} \hat{a}_{\xi} X+\hat{\nabla}_{X}^{\perp} \xi,
$$

for any $X \in \chi(M), \xi \in \Gamma^{\infty}\left(\mathcal{\nu}^{2 k}(f)\right)$, we find

$$
\begin{aligned}
& \hat{\nabla}_{T_{\beta}}^{\perp} \xi_{a}=\nabla_{\bar{T}_{\beta}}^{\perp} \xi_{a}+2 u_{\beta} \zeta_{a}, \\
& \hat{\nabla}_{T_{\bar{\beta}}}^{\perp} \zeta_{a}=\nabla_{T_{\bar{\beta}}}^{\perp} \zeta_{a}, \\
& \hat{\nabla}_{\hat{T}^{\perp} \zeta_{a}}=\nabla_{\hat{T}^{\perp}}^{\perp} \zeta_{a}+2 u_{0} e^{-2 u} \zeta_{a} .
\end{aligned}
$$

If $M=H_{n}$ and $A=H_{N}$ we have $\nabla^{A} \zeta_{a}=0$ and thus $\nabla^{\perp} \zeta_{a}=0$. Thus (by (74)) if $M=H_{n}(s)$ and $A=H_{N}(s)$ the normal Tanaka-Webster connection of $F$ is given by

$$
\nabla_{T_{\beta}}^{1} \zeta_{a}=2 u_{\beta} \zeta_{a},
$$

$$
\begin{aligned}
& \nabla_{T}^{\perp} \zeta_{a}=0, \\
& \nabla_{T}^{\frac{1}{T}} \zeta_{a}=2 u_{0} e^{-2 u} \zeta_{a},
\end{aligned}
$$

with $u=\log |x|^{-1}$. Next (as a consequence of (75)) we may use the identities

$$
\begin{aligned}
& {\left[T_{\alpha}, T_{\beta}\right]=0,} \\
& {\left[T_{\alpha}, T_{\bar{\beta}}\right]=-2 i \delta_{\alpha \beta} \frac{\partial}{\partial t},}
\end{aligned}
$$

and

so that to yield

$$
\nabla_{\partial / \partial t}^{\perp} \zeta_{a}=2\left(u_{0}+2 i u_{\beta} u^{\beta}\right) \zeta_{a}
$$

$$
R^{\perp}\left(T_{\alpha}, T_{\beta}\right) \zeta_{a}=0, \quad R^{\perp}\left(T_{\bar{\alpha}}, T_{\bar{\beta}}\right) \zeta_{a}=0,
$$

and

$$
R^{\perp}\left(T_{\alpha}, T_{\bar{\beta}}\right) \zeta_{a}=\left\{-2 T_{\bar{\beta}}\left(u_{\alpha}\right)+4 i \delta_{\alpha \beta}\left(u_{0}+2 i u_{\sigma} u^{\sigma}\right)\right\} \zeta_{a} .
$$

Finally, taking into account the identities 
it follows that

$$
\begin{aligned}
& u_{\alpha}=-\frac{1}{2}|x|^{-4} \bar{z}_{\alpha} \phi, \quad T_{\bar{\beta}}\left(u_{\alpha}\right)=-\frac{1}{2}|x|^{-4} \delta_{\alpha \beta} \phi, \\
& u_{0}=-\frac{1}{2}|x|^{-4} t, \quad \phi \bar{\phi}=|x|^{4}, \\
& u_{\sigma} u^{\sigma}=\frac{1}{4}|x|^{-4}|z|^{2},
\end{aligned}
$$

$$
R^{\perp}\left(T_{\alpha}, T_{\bar{\beta}}\right) \zeta_{a}=-|x|^{-4} \phi \delta_{\alpha \beta} \zeta_{a} .
$$

Summing up, the pseudohermitian immersion $F: H_{n}(s) \rightarrow H_{N}(s)$ has (by $\left.(77)\right) R^{\perp} \neq 0$. However (55) yields $K_{\alpha \bar{\beta}}=\lambda R_{\alpha \bar{\beta}}$ with $\lambda=(N+1) /(n+1)$.

\section{3) (Pseudo-Siegel domains)}

Let $(\alpha, \beta)=\left(\alpha_{1}, \cdots, \alpha_{n}, \beta\right) \in Z_{+}^{n+1}$ be a fixed multiindex and $D_{\alpha, \beta}=\left\{\left(z_{1}, \cdots\right.\right.$, $\left.\left.z_{n}, w\right) \in C^{n+1}: \sum_{j=1}^{n}\left|z_{j}\right|^{2 \alpha_{j}}+\operatorname{Im}\left(w^{\beta}\right)-1<0\right\} \quad$ (cf. [BP]). Then $D_{1,1}$ is the Siegel domain in $C^{n+1}$ (and $\partial D_{1,1} \approx H_{n}$ ). Assume $\beta>1$ from now on. The boundary $\partial D_{\alpha, \beta}$ of $D_{\alpha, \beta}$ inherits a CR structure (as a real hypersurface of $C^{n+1}$ ) spanned by

$$
T_{\jmath}=\frac{\partial}{\partial z_{\jmath}}-2 i f_{j} \frac{\partial}{\partial w},
$$

in some neighborhood of $w \neq 0$ where

$$
f_{\jmath}=\frac{\alpha \jmath}{\beta} w^{1-\beta} z_{j}^{\alpha_{j}^{-1}} \bar{z}_{j}^{\alpha}
$$

Hence we have the commutation relations

$$
\begin{gathered}
{\left[T_{j}, T_{k}\right]=0,} \\
{\left[T_{\jmath}, T_{\bar{k}}\right]=\frac{2 i}{\beta}\left\{\frac{\alpha_{\jmath}^{2}\left|z_{\jmath}\right|^{2\left(\alpha_{j}-1\right)}}{w^{\beta-1}} \frac{\partial}{\partial w}+\frac{\alpha_{k}^{2}\left|z_{k}\right|^{2\left(\alpha_{k}-1\right)}}{\bar{w}^{\beta-1}} \frac{\partial}{\partial \bar{w}}\right\} \delta_{j k} .}
\end{gathered}
$$

Endow $\partial D_{\alpha, \beta}$ with the pseudohermitian structure $\theta=\theta_{\alpha, \beta}$ given by

$$
\theta=\beta w^{\beta-1} d w+\beta \bar{w}^{\beta-1} d \bar{w}+2 i \sum_{j=1}^{n}\left(g_{j} d z_{j}-\bar{g}_{j} d \bar{z}_{j}\right),
$$

where

$$
g_{\jmath}=\alpha_{j} z_{\jmath}^{\alpha_{j}-1} \bar{z}_{\jmath}^{\alpha}
$$

Therefore the Levi form of $\left(\partial D_{\alpha, \beta}, \theta\right)$ is $\operatorname{diag}\left(\lambda_{1}, \cdots, \lambda_{n}\right)$ where

$$
\lambda_{\jmath}=4 \alpha_{j}^{2}\left|z_{j}\right|^{2\left(\alpha_{j}-1\right)} \text {. }
$$

Therefore, if $\alpha_{\jmath}>1,1 \leqq j \leqq n$, then $G_{\theta}$ is degenerate at each point of $\bigcup_{\jmath=1}^{n} M_{\jmath}$, where $M_{j}$ is the trace of the complex hyperplane $L_{j}=\left\{(z, w): z_{j}=0\right\}$ on the boundary of $D_{\alpha, \beta}$. Next $U_{\alpha, \beta}=\partial D_{\alpha, \beta}-\left(\cup_{j=1}^{n} M_{j}\right.$ ) (an open subset of $\partial D_{\alpha, \beta}$ ) is a strictly pseudoconvex CR manifold. The characteristic direction $T$ of 
is given by

$$
d \theta=-4 i \alpha_{\jmath}^{2}\left|z_{\jmath}\right|^{2\left(\alpha_{j}-1\right)} d z_{\jmath} \wedge d \bar{z}^{\jmath}
$$

$$
T=\frac{1}{4 \beta|w|^{2(\beta-1)}}\left\{\bar{w}^{\beta-1} \frac{\partial}{\partial w}+w^{\beta-1} \frac{\partial}{\partial \bar{w}}\right\}
$$

Note that (79) may be written

Also

$$
\begin{aligned}
& \jmath \neq k \Longrightarrow\left[T_{\jmath}, T_{\bar{k}}\right]=0, \\
& {\left[T_{\jmath}, T_{j}\right]=i \lambda_{\jmath} T .}
\end{aligned}
$$

$$
[T, T]=0 \text {. }
$$

Using (A.3)-(A.5) in [D2], p. 48, we derive the (Christoffel symbols of the) Tanaka-Webster connection of $\left(U_{\alpha, \beta}, \theta\right)$

$$
\Gamma_{j k}^{s}=\frac{\alpha_{j}-1}{z_{\jmath}} \delta_{j k} \delta_{j s}, \quad \Gamma_{j k}^{s}=0, \quad \Gamma_{0 k}^{s}=0 .
$$

Therefore $\left(U_{\alpha, \beta}, \theta\right)$ has a vanishing pseudohermitian torsion $(\tau=0)$. As a straightforward consequence of (81) the Tanaka-Webster connection of $\left(U_{\alpha, \beta}, \theta\right)$ is flat $(R=0)$.

Finally we look at the structure of the points of weak pseudoconvexity of $\partial D_{\alpha, \beta}$. Let $1 \leqq p \leqq n$ and set $M_{\jmath_{1} \cdots \jmath_{p}}=\partial D_{\alpha, \beta} \cap L_{\jmath_{1}} \cap \cdots \cap L_{\jmath_{p}}$. Then

$$
M_{\jmath_{1} \cdots \jmath_{p}} \approx \partial D_{\alpha \jmath_{1} \cdots \jmath_{p}, \beta} \subset C^{n+1-p},
$$

(a diffeomorphism), where $\alpha_{\rho_{1} \cdots j_{p}}=\left(\alpha_{1}, \cdots, \hat{\alpha}_{\rho_{1}}, \cdots, \hat{\alpha}_{\rho_{p}}, \cdots, \alpha_{n}\right)$. A natural question is how does $M_{\jmath_{1} \cdots j_{p}}$ sit in $\partial D_{\alpha, \beta}$ i.e. equivalently study the geometry of the immersion $f: \partial D_{\left(\alpha_{1}, \cdots, \alpha_{k}\right), \beta} \rightarrow \partial D_{\alpha, \beta}$ induced by the natural map $\boldsymbol{C}^{k} \times \boldsymbol{C} \rightarrow \boldsymbol{C}^{n} \times \boldsymbol{C}$, $(z, w) \mapsto(z, 0, w), 0 \in \boldsymbol{C}^{k}, 0<k<n$. Using (78) one may show that $f$ is a $\mathrm{CR}$ immersion. Finally (80) yields $f * \theta_{\alpha, \beta}=\theta_{\left(\alpha_{1}, \ldots, \alpha_{k}\right), \beta}$ i.e. $f$ is isopseudohermitian.

\section{REFERENCES}

[A] T. AKAHORI, A new approach to the local embedding theorem of CR structures, the local embedding theorem for $n \geqq 4$, Mem. Amer. Math. Soc., 366 (1987).

[BP] E. BARletTA AND C. PARRINI, Bounded solutions for the $\bar{\partial}$-problem in pseudoSiegel domains, Ann. Mat. Pura Appl., 168 (1995), 119-132.

[B] D.E. BLAIR, Contact manifolds in Riemannian geometry, Lecture Notes in Math., 509, Springer-Verlag, Berlin-Heidelberg-New York, 1976.

[BM] L. Boutet DE Monvel, Intégration des équations de Cauchy-Riemann induites formelles, Sém. Goulaouic-Lions-Schwartz (1974-75), Centre Math. École Politech., Paris, 1975.

[CL] B. Y. CHEN AND H.S. LUE, On normal connection of Kähler submanifolds, J. Math. Soc. Japan, 27 (1975), 550-556.

[D1] S. DRAGOMIR, On pseudohermitian immersions between strictly pseudoconvex CR manifolds, Amer. J. Math., 117 (1995), 169-202. 
[D2] S. DRAGomir, On a conjecture of J. M. Lee, Hokkaido Math. J., 23 (1994), 35-49.

[D3] S. DRAGomiR, Generalized Hopf manifolds, locally conformal Kähler structures, and real hypersurfaces, Kodai Math. J., 14 (1991), 366-391.

[FS] G.B. Folland AND E.M. SteIN, Estimates for the $\bar{\partial}_{b}$-complex and analysis on the Heisenberg group, Comm. Pure Appl. Math., 27 (1974), 429-522.

[G] S. Golberg, Curvature and Homology, Dover Publ., New York, 1982.

[J] H. JAcoBowitz, Mappings between CR manifolds, Recent Developments in Several Complex Variables, Ann. of Math. Studies, 100, Princeton Univ. Press, Princeton, 1981, 227-241.

[JL1] D. JERISON AND J.M. LEe, The Yamabe problem on CR manifolds, J. Differential Geom., 25 (1987), 167-197.

[JL2] D. JERISON AND J.M. LEE, CR normal coodinates and the CR Yamabe problem, J. Differential Geom., 29 (1989), 303-344.

[KN] S. Kobayashi AND K. NomizU, Foundations of Differential Geometry, Interscience Publ., New York, vol. I, 1963, vol. II, 1969.

[K] M. KuRAnishi, Strongly pseudoconvex CR structures over small balls, I, Ann. of Math., 115 (1982), 451-500, II, ibid., 116 (1982), 1-64, III, ibid., 116 (1982), 249-330.

[L1] J.M. LEE, Pseudo-Einstein structures on CR manifolds, Amer. J. Math., 110 (1998), 157-178.

[L2] J.M. LEE, The Fefferman metric and pseudohermitian invariants, Trans. Amer. Math. Soc., 296 (1986), 411-429.

[T] N. Tanaka, A Differential Geometric Study on Strictly Pseudoconvex Manifolds, Kinokuniya Company Ltd., Tokyo, 1975.

[U1] H. URAKAWA, Yang-Mills connections over compact strongly pseudoconvex CR manifolds, Math. Z., 216 (1994), 541-573.

[U2] H. URAKAwA, Yang-Mills connections and deformation theory over compact strongly pseudoconvex $C R$ manifolds, To appear in Proceedings of the third MSJ International Research Institute on Geometric Complex Analysis, held at Hayama, Japan, March 19-29, 1995, World Scientific, 1995.

[U3] H. URAKAwA, Variational problems over strongly pseudoconvex $\mathrm{CR}$ manifolds, Differential Geometry, Editors C.H. Gu, H.S. Ho and Y.L. Xin, World Scientific, Singapore-New Jersey-London-Hong Kong, 1993, 233-242.

[W1] S. Webster, Pseudohermitian structures on a real hypersurface, J. Differential Geom., 13 (1978), 25-41.

[W2] S. WEBSTER, The rigidity of CR hypersurfaces in a sphere, Indiana Univ. Math. J., 28 (1979), 405-416.

\author{
Università della Basilicata \\ Dipartimento di MATEMATICA \\ VIA N. SAURO 85 \\ Potenza, Italy \\ Politecnico di Milano \\ Dipartimento di Matematica \\ Piazza L. DA Vinci 32 \\ Milano, Italy
}

